\author{
ABSTRACT \\ RESONANT EXTRACTION FOR MU2E \\ Prudhvi Raj Varma Chintalapati, M.S. \\ Department of Physics \\ Northern Illinois University, 2019 \\ Michael J. Syphers, Director
}

Charged Lepton Flavor Violations (CLFVs) are probed in an attempt to search Beyond Standard Model (BSM) Physics. The sensitive channel available for experiments around the world is to probe through muons. No conclusive evidence of CLFV is found so far but Mu2e experiment at Fermi National Accelerator Laboratory (Fermilab) aims to improve the current experimental sensitivity by searching for $\mu^{-} N \longrightarrow e^{-} N$ transition. To provide the high intensity beam that can be handled to achieve desired sensitivity, slow spill using resonant extraction is used at the accelerator complex.

Understanding the motivations behind the experiment and simulating the crucial stage in the beam delivery system is the main focus of this thesis. Work presented here will have a qualitative analysis and simulation of resonant extraction at the Delivery Ring (Fermilab), using the beam parameters of $M u 2 e$ to study various factors that contribute to the successful beam transport towards the Target and Detector System, including meeting intensity requirements while keeping the beam losses to a minimum. 
NORTHERN ILLINOIS UNIVERSITY

DE KALB, ILLINOIS

JUNE 2019

\title{
RESONANT EXTRACTION FOR MU2E
}

\author{
BY \\ PRUDHVI RAJ VARMA CHINTALAPATI \\ (C) 2019 Prudhvi Raj Varma Chintalapati
}

\begin{abstract}
A THESIS SUBMITTED TO THE GRADUATE SCHOOL IN PARTIAL FULFILLMENT OF THE REQUIREMENTS

FOR THE DEGREE

MASTER OF SCIENE
\end{abstract}

DEPARTMENT OF PHYSICS

Thesis Director:

Michael J. Syphers 


\section{ACKNOWLEDGEMENTS}

The author would like to acknowledge the guidance and support from Michael Syphers and Stephen Martin, who provided the amazing opportunity of working with them and were patient throughout my learning process. I would like to thank Diktys Stratakis for his insights and assistance that provided a great deal of encouragement for my work. I would also like to thank our research group for their constant exchange of ideas.

I would like to thank John Johnstone for kindly sharing his MAD lattice file for the delivery ring which has been used as the central part of my simulations. I would also like to thank Vladimir Nagaslav for his generosity in sharing the key parameters being used for resonant extraction in $M u 2 e$.

Particle tracking simulations were created using Gaea cluster at Northern Illinois University (NIU) and results were obtained in a reasonable amount of time because of the great support from the staff of NIU's Center for Research Computing and Data.

Finally I would like to thank Nathan Froemming and Christina Sarosiek who proved to be amazing reviewers providing valuable suggestions for editing my thesis, and helping me present my work in a professional way to the reader.

This work is supported by Physics Department at Northern Illinois University. A portion of the work was performed at Fermi National Accelerator Laboratory (Fermilab), a U.S. Department of Energy, Office of Science, HEP User Facility. Fermilab is managed by Fermi Research Alliance, LLC (FRA), acting under Contract No. DE-AC02-07CH11359. 


\section{DEDICATION}

For their belief and support, which allowed me to reach one of the most important and memorable milestones in my career, I would like to dedicate this work to Amma, Baba, Sowji, Raji, Varma, Rishi, Bharathi, Andrew, Rooms and Praveen. 


\section{TABLE OF CONTENTS}

Page

List of Tables $\ldots \ldots \ldots \ldots \ldots \ldots \ldots \ldots \ldots \ldots \ldots \ldots \ldots \ldots \ldots$ vii

List of Figures $\ldots \ldots \ldots \ldots \ldots \ldots \ldots \ldots \ldots \ldots \ldots \ldots \ldots \ldots \ldots$ viii

List of Appendices . . . . . . . . . . . . . . . . . . . $\quad \mathrm{x}$

Chapter

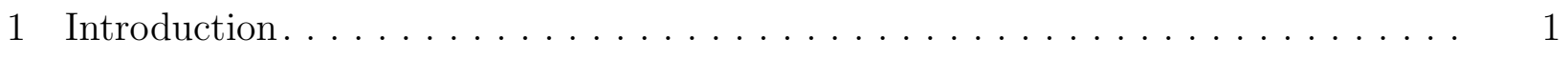

1.1 What is Charged Lepton Flavor Violation (CLFV)?. . . . . . . . . . . . . 2

1.2 Theoretical Predictions of CLFV $\ldots \ldots \ldots \ldots \ldots \ldots \ldots$

1.2.1 Extensions to Standard Model [6] [7] . . . . . . . . . . . . . . . 3

1.2 .2 CLFV in Supersymmetry (SUSY) [6] [7][8]. . . . . . . . . . . . . . 5

1.3 Experiments in search for CLFV. . . . . . . . . . . . . . 6

$1.4 \quad M u 2 e$ at Fermilab $[2][19] \ldots \ldots \ldots \ldots \ldots \ldots \ldots$

1.4 .1 Beam Transport . . . . . . . . . . . . . . . . . . 9

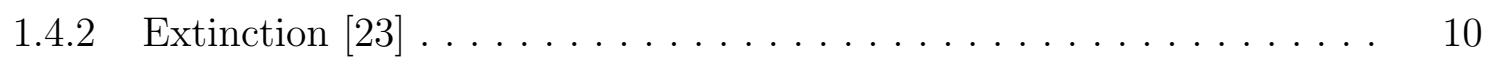

1.4 .3 Target and Detector system [24] . . . . . . . . . . . . . 11

2 Accelerator Physics. . . . . . . . . . . . . . . . . . . . . 14

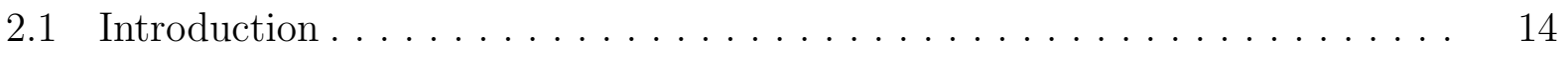

2.2 Charged Particle in a Electromagnetic Field . . . . . . . . . . . . . . . 15

2.3 Coordinates in accelerator systems $\ldots \ldots \ldots \ldots \ldots$

2.3.1 Revisiting charged particle in an Electromagnetic Field . . . . . . . . 18 
Chapter Page

2.4 Magnets. . . . . . . . . . . . . . . . . . . . . . . . 20

2.4 .1 Dipole . . . . . . . . . . . . . . . . . . . 22

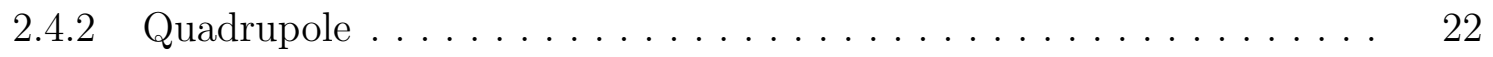

2.4 .3 Sextupole. . . . . . . . . . . . . . . . . . . . . 24

2.5 Courant-Snyder Parameters [32] . . . . . . . . . . . . . . . 25

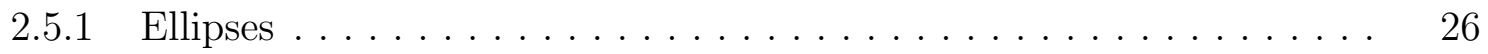

$2.5 .2 \quad$ Normalized Phase Space . . . . . . . . . . . . . . . . . 28

2.6 Resonance ................................ 29

2.6 .1 Dipole Resonance. . . . . . . . . . . . . . . . . . . 29

2.6.2 Quadrupole and Sextupole Resonances. . . . . . . . . . . . . . . 30

2.7 Resonant Extraction [26] . . . . . . . . . . . . . . . . . 31

2.7.1 Non-linear Resonance using Sextupoles. . . . . . . . . . . . . . . 33

2.7 .2 Equations of motion for sextupole resonance. . . . . . . . . . . 34

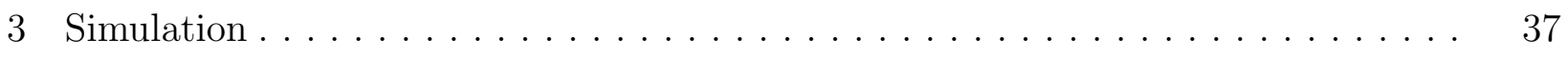

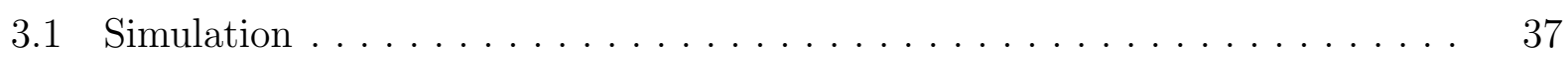

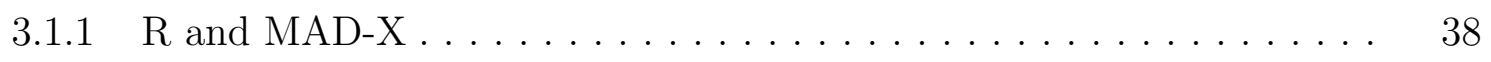

3.2 Setup and Parameters . . . . . . . . . . . . . . . . . . 38

3.3 Third Integer Resonance . . . . . . . . . . . . . . . . . . . . . 40

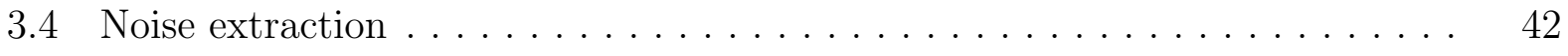

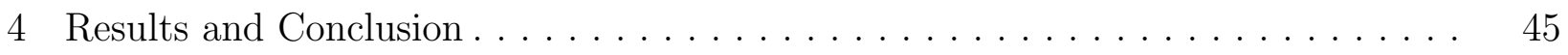

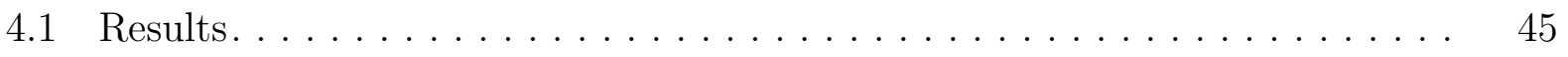

4.1 .1 Interpreting Results . . . . . . . . . . . . . . . . 46

4.2 Future directions . . . . . . . . . . . . . . . . . 51

4.3 Conclusions .......................... 51 
Chapter $\quad$ Page

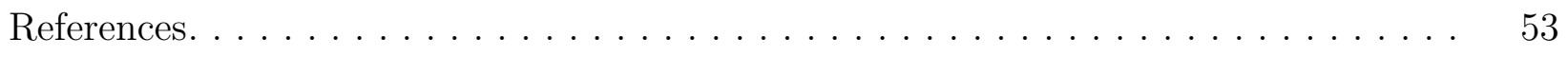

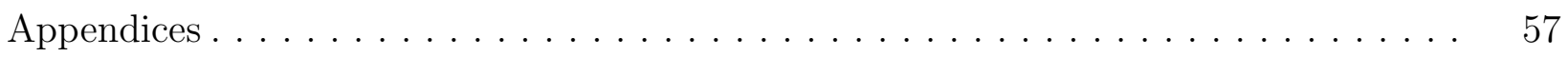




\section{LIST OF TABLES}

Table $\quad$ Page

1.1 Particles that would be relevant in CLFV [6] . . . . . . . . . . . . 5

3.1 Parameters that are considered for this simulation [2]. . . . . . . . 40

3.2 Parameters that are considered for extraction [2] . . . . . . . . . . . . 42 


\section{LIST OF FIGURES}

Figure

Page

1.1 Standard Model of Elementary Particles [3] . . . . . . . . . . . . . . . 2

$1.2 \mu \longrightarrow e \gamma$, momenta of the particles is given in parenthesis (SM with massive

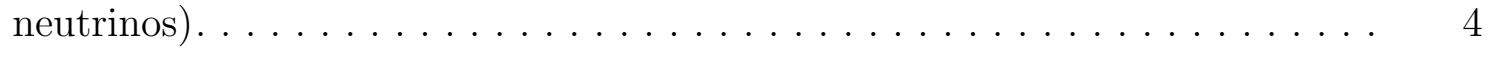

1.3 Example contributions to $\mu \longrightarrow e \gamma$. Left to Right: Bino contribution, Wino contribution and Higgsino Contribution [10] $\ldots \ldots \ldots \ldots \ldots$

1.4 Example contributions to $\mu^{-} N \longrightarrow e^{-} N[10] \ldots \ldots \ldots \ldots$

1.5 Limit on the branching ratio of flavor violation set by experiment as a func-

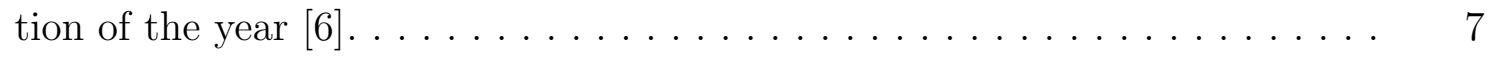

1.6 Fermilab Accelerator Campus [22] . . . . . . . . . . . . . . . . 9

1.7 Muon Campus ................................ 10

1.8 Production, Transport and Detector Solenoid systems [25] . . . . . . . . . 11

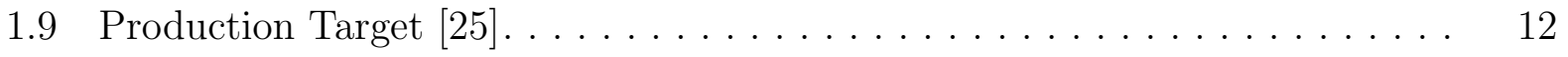

2.1 Coordinates in Particle Accelerators . . . . . . . . . . . . . . . . 17

2.2 Dipole Magnet [29]. . . . . . . . . . . . . . . . . . . . . . . 20

2.3 Quadrupole Magnets [30]. . . . . . . . . . . . . . . . . . . . . . 20

2.4 Sextupole[31] . . . . . . . . . . . . . . . . . . . . 24

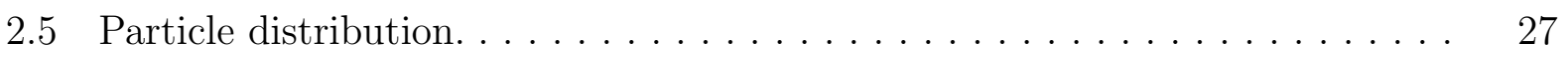

2.6 Phase space mapping. . . . . . . . . . . . . . . . . . . . . . 27

2.7 Normalized Particle distribution. . . . . . . . . . . . . . . . . . . . 29

2.8 Normalized Phase Space. . . . . . . . . . . . . . . . . . . . . . . 29

2.9 Dipole Resonance. . . . . . . . . . . . . . . . . . . . . . . 30 
Figure $\quad$ Page

2.10 Quadrupole Resonance. . . . . . . . . . . . . . . . . . . . . . . . . 30

2.11 Sextupole Resonance. . . . . . . . . . . . . . . . . . . . . . . . . . . 31

2.12 Separatrix for sextupole resonance . . . . . . . . . . . . . . . . 36

2.13 Two sextupoles . . . . . . . . . . . . . . . . . . . 36

3.1 Delivery Ring (DR) Lattice[37] and amplitude function of $\beta_{x}$ and $\beta_{y}$ for each

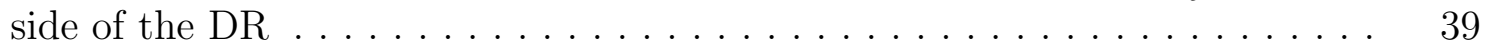

3.2 Sextupoles Placement . . . . . . . . . . . . . . . . . . . . 41

3.3 Phase evolution in R (on the Left) and MAD-X (on the Right) . . . . . . . . 42

3.4 Noise introduction in Phase space . . . . . . . . . . . . . . . . . 43

3.5 Emittance change due to noise . . . . . . . . . . . . . . . . 44

4.1 Tune 0-250; Sextupole 0-500; Noise $\left(\Delta x^{\prime}\right)=10^{-6} \ldots \ldots \ldots \ldots$. . . . . 47

4.2 Tune 0-250; Sextupole 0-500; Noise $\left(\Delta x^{\prime}\right)=5 \times 10^{-6} \ldots \ldots \ldots \ldots$. . . . . 48

4.3 Tune 0-250; Sextupole 0-500; Noise $\left(\Delta x^{\prime}\right)=8 \times 10^{-6} \ldots \ldots \ldots$. . . . . . 48

4.4 Tune 0-250; Sextupole 0-500; Noise $\left(\Delta x^{\prime}\right)=2 \times 10^{-6} \ldots \ldots \ldots \ldots$. . . . . 49

4.5 Tune 0-1000; Sextupole 0-1000; Noise $\left(\Delta x^{\prime}\right)=2 \times 10^{-6} \ldots \ldots \ldots$. . . . . . 49

4.6 Tune 0-2000; Sextupole 0-2000; Noise $\left(\Delta x^{\prime}\right)=2 \times 10^{-6} \ldots \ldots \ldots$. . . . . 50 


\section{LIST OF APPENDICES}

Appendix $\quad$ Page

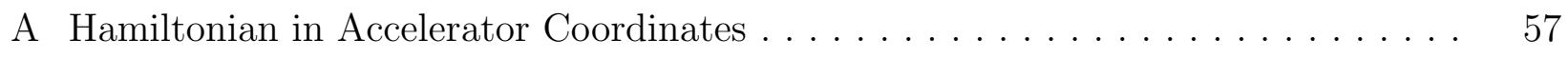

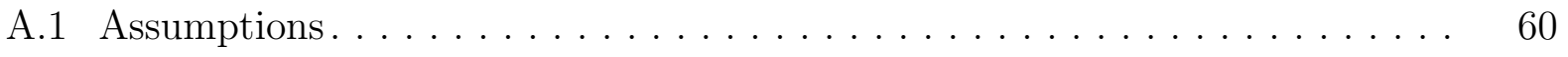

B Matrix representation of Courant-Snyder parameters . . . . . . . . . . . . . . . 62

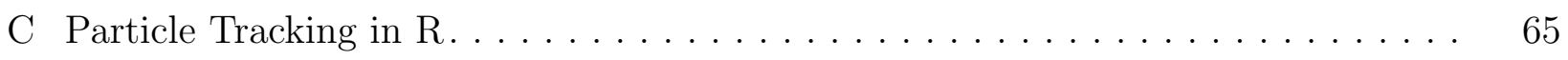




\section{CHAPTER 1 INTRODUCTION}

The Standard Model (SM) in particle physics is a widely accepted theory which attempts to explain the fundamentals of how everything in the Universe works. It is widely accepted because of its huge success in predicting the existence of fundamental particles and their interactions before they are observed and confirmed in experiments (the theory's latest discovery being Higgs boson in 2012 [1]).

Despite its success, the theory has gaps, such as being unable to explain gravity, the hierarchy problem, dark matter, dark energy, etc. Several theories have been proposed, studied, and tested in attempts to explain what the standard model couldn't, but none have had the same level of success as the SM. Experiments are being designed to generate events which can either contradict SM, predict a theory beyond Standard Model (BSM), or both simultaneously to find answers we don't currently have. Mu2e[2] is one such experiment under construction now at Fermi National Accelerator Laboratory (Fermilab). This experiment is looking for Charged Lepton Flavor Violation (CLFV) transitions which violate flavor physics in the SM and are a way to look for physics BSM.

In the following sections we will learn about CLFV, theoretical predictions, current ex-

perimental limits and the purpose of $M u 2 e$. Then we will discuss the experimental setup of Mu2e at Fermilab before finally discussing one of the key techniques that would make the experiment a reality at Fermilab.

Chapter two will discuss in detail accelerator physics and the final chapter will present the simulation, results, analysis, and conclusion. 


\section{Standard Model of Elementary Particles}

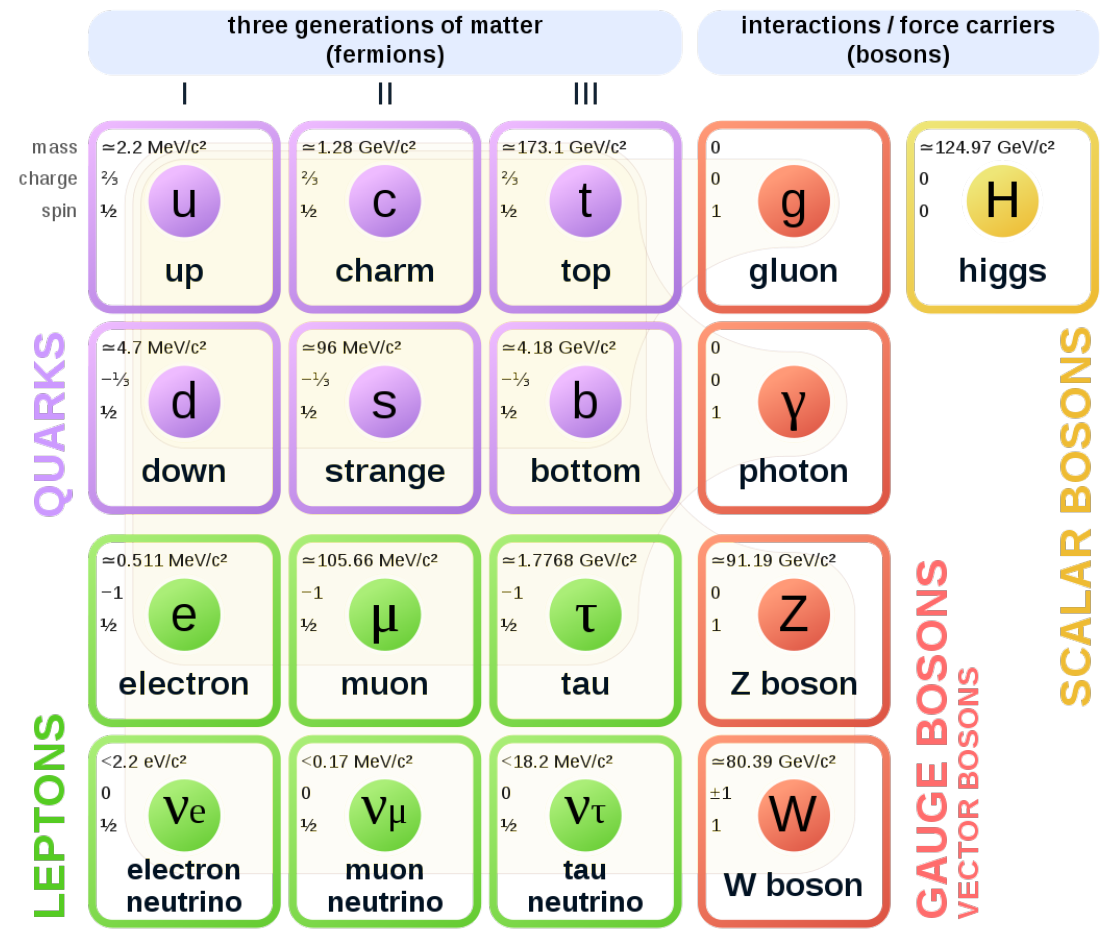

Figure 1.1: Standard Model of Elementary Particles [3]

\subsection{What is Charged Lepton Flavor Violation (CLFV)?}

The Standard model (SM) consists of two basic building blocks: fermions (matter particles) and bosons (force carriers). Fermions are further classified into two groups, quarks and leptons, of which quarks can interact through strong force while leptons can't. The SM of elementary particles is shown in Figure 1.1.

The electron, the muon and the tau are three different flavored charged leptons. Charged Lepton Flavor Violation is a neutrino-less transition where one flavor charged lepton is converted to another. The SM doesn't predict CLFV so the observation of any such event would lead to new physics beyond the standard model. 
Half a century ago there was a solar neutrino problem which stymied astrophysics. The Sun's fusion process produces a large number of electron neutrinos; however, in the 1960s detectors measured the influx of solar neutrinos at a lower number than predicted by the SM. Results from the Super-Kamiokande detector later confirmed that these observations are a result of neutrino oscillations. This confirmation implies that neutrinos have mass and also shows evidence of flavor changing between leptons [4]. This detection of Lepton Flavor Violation led to a discussion on the possibility of Charged Lepton Flavor Violation (CLFV).

\subsection{Theoretical Predictions of CLFV}

Because the availability of muons in accelerators and cosmic radiation is abundant, three rare processes are being looked into globally to detect CLFV. They are ideal candidates because their sensitivities are within the experimental limitations. They are:

$$
\mu^{+} \longrightarrow e^{+} \gamma, \quad \mu^{-} N \longrightarrow e^{-} N, \quad \mu^{+} \longrightarrow e^{+} e^{-} e^{+}
$$

We will start with discussion of how extensions to SM predict CLFV before moving to other theories with more deviations from SM.

\subsubsection{Extensions to Standard Model [6] [7]}

Neutrino oscillations encourage an extension of the SM by including neutrino mass terms. To accommodate this we can introduce right-handed neutrinos by taking the same Diracmass framework that has been used for other fermions. However, for this theory to work, Yukawa couplings have to be extremely small (no larger than $10^{-9}$ ) giving rise to another, 
more popular theory called the seesaw mechanism. The seesaw mechanism suggests that the small masses of neutrinos naturally occur as a result of introducing Majorana fermion fields and a corresponding mass matrix whose magnitude is large compared to the Dirac mass matrix. This theory predicts three extremely heavy mass eigenstates for neutrinos, in addition to the three SM states (where the lighter ones are Majorana fermions). Neutrino oscillations can result in CLFV only through loop diagrams, an example of which is shown in Figure 1.2. For the example $\mu \longrightarrow e \gamma$ the branching ratio is of the order $10^{-54}$ which is out of current experimental reach.

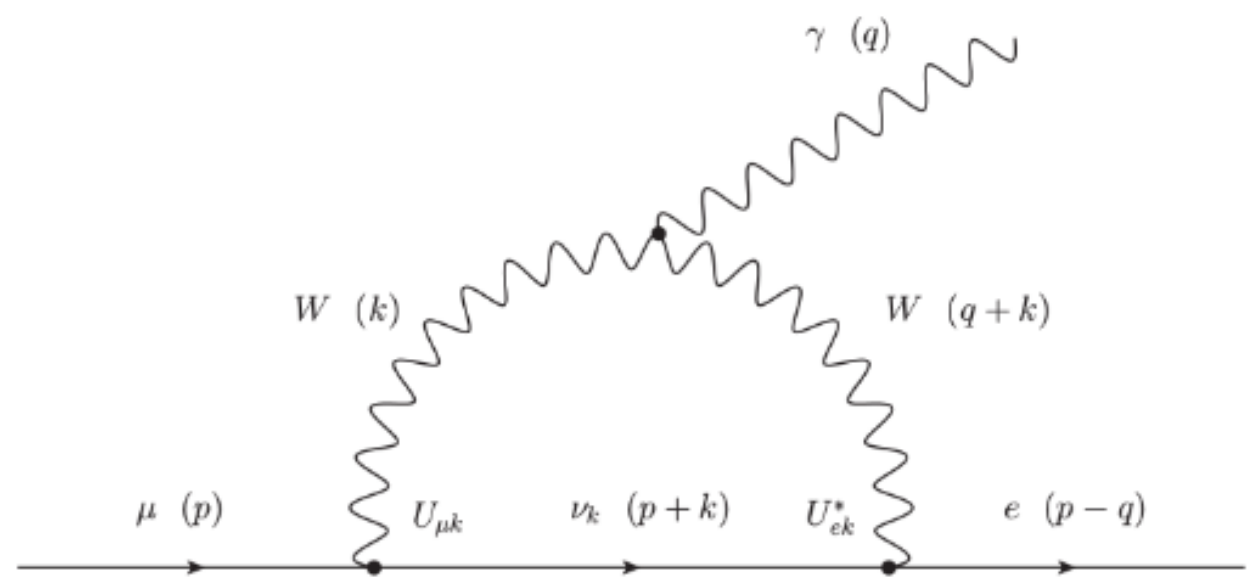

Figure 1.2: $\mu \longrightarrow e \gamma$, momenta of the particles is given in parenthesis (SM with massive neutrinos)

Another approach is based on the Higgs field. Different flavors in the lepton family are a result of the Yukawa couplings of fermion fields with the Higgs scalar field, $\Phi$, and its vacuum expectation value (vev). Many theories suggest that there exists two Higgs fields which means the mass of leptons depends on two vevs and more coupling constants. These theories produce flavor changing neutral currents (FCNC) that generate CLFV at the tree level, unlike earlier case where there is CLFV at the loop level. 


\subsubsection{CLFV in Supersymmetry (SUSY) [6][7][8]}

The hierarchy problem of the SM is one of the main motivations for the development of SUSY. In order to fine-tune the quantum loop corrections for the higgs mass, SUSY builds a mathematical framework that suggests the existence of a superpartner for each existing particle in the SM. This means that each fermion has boson as a superpartner and vice versa. The particle along with its superpartner together form a supermultiplet. By assuming the existence of supermultiplets, quantum loop corrections to Higgs mass parameter would vanish because of the relative negative sign between the fermion and boson quantum loop corrections.

Table 1.1: Particles that would be relevant in CLFV [6]

\begin{tabular}{|c||c|}
\hline Gauge Eigenstates & SM partners \\
\hline \hline$\tilde{B}, \tilde{W}^{0}, \tilde{H}_{u}^{0}, \tilde{H}_{d}^{0}$ & $\gamma, Z, h$ \\
Bino, neut. Wino and Higgsinos & \\
\hline$\tilde{W}^{ \pm}, \tilde{H}_{u}^{+}, \tilde{H}_{d}^{-}$ & $W^{ \pm}$ \\
charg. Wino and Higgsinos & \\
\hline$\tilde{e}^{ \pm}, \tilde{\mu}^{ \pm}, \tilde{\tau}^{ \pm}$ & $e^{ \pm}, \mu^{ \pm}, \tau^{ \pm}$ \\
$\tilde{\nu}_{e}, \tilde{\nu}_{\mu}, \tilde{\nu_{\tau}}$ & $\nu_{e}, \nu_{\mu}, \nu_{\tau}$ \\
RH/LH selectron, smuon, stau & \\
\hline
\end{tabular}

Theories like PeV scale SUSY[9] show no compelling deviation from SM disallowing FCNC and CLFV. Other SUSY theories do allow CLFV with sensitivities ranging from very small to present experimental limits. Table 1.1 shows the particles and their superpartners from SUSY relevant to CLFV. In addition, the correspondences between SM particles and 
their SUSY partners are shown in the table. The couplings between the pairs result in CLFVs. A few example contributions to $\mu \longrightarrow e \gamma$ are shown in Figures 1.3 and 1.4. The detailed analysis of these contributions can be found in [10].
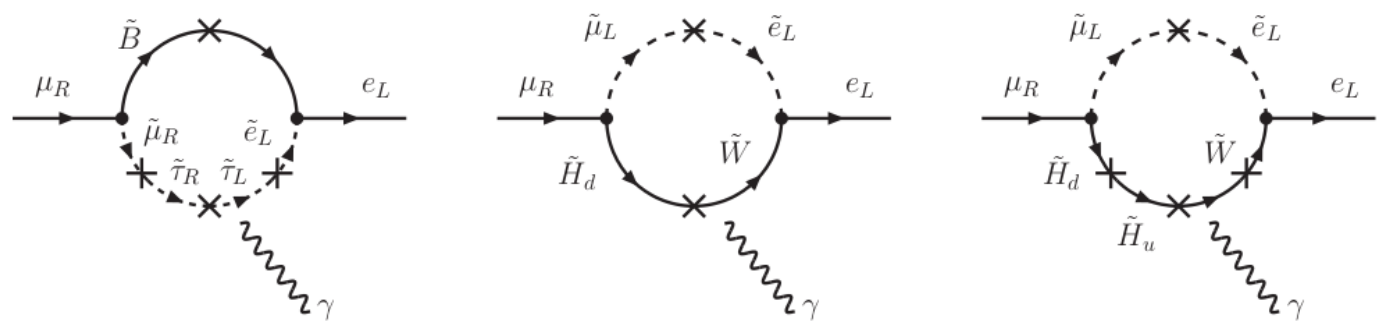

Figure 1.3: Example contributions to $\mu \longrightarrow e \gamma$. Left to Right: Bino contribution, Wino contribution and Higgsino Contribution [10]
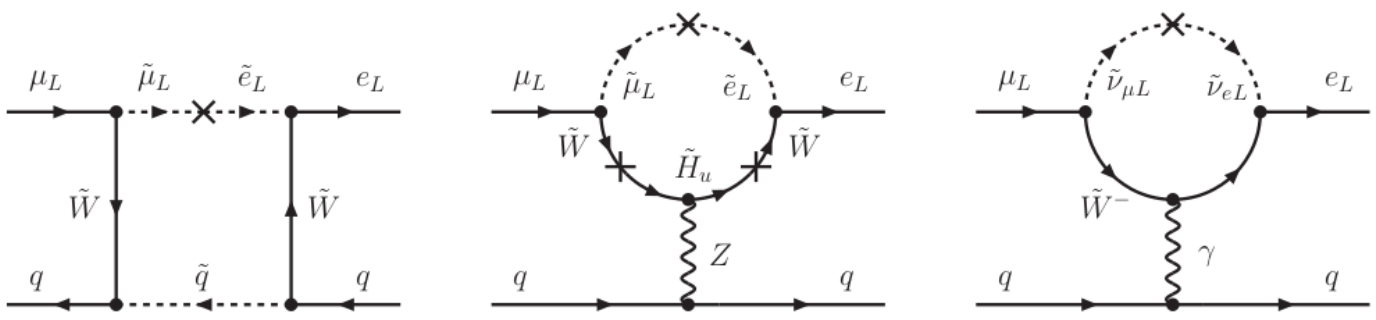

Figure 1.4: Example contributions to $\mu^{-} N \longrightarrow e^{-} N$ [10]

Rigorous details of various SUSY models can be found here: [6], [8], [9], [10], [11], [12] [13].

\subsection{Experiments in search for CLFV.}

Historically, the search for CLFV is a popular choice among the experiments looking for BSM Physics. Continous efforts have been undertaken to increase the sensitivity limits on CLFV. Experimental limits have come a long way in last 50 years as it can be seen in 
Figure 1.5. So far no evidence of CLFV has been found, but physicists are still hopeful enough to look for it.

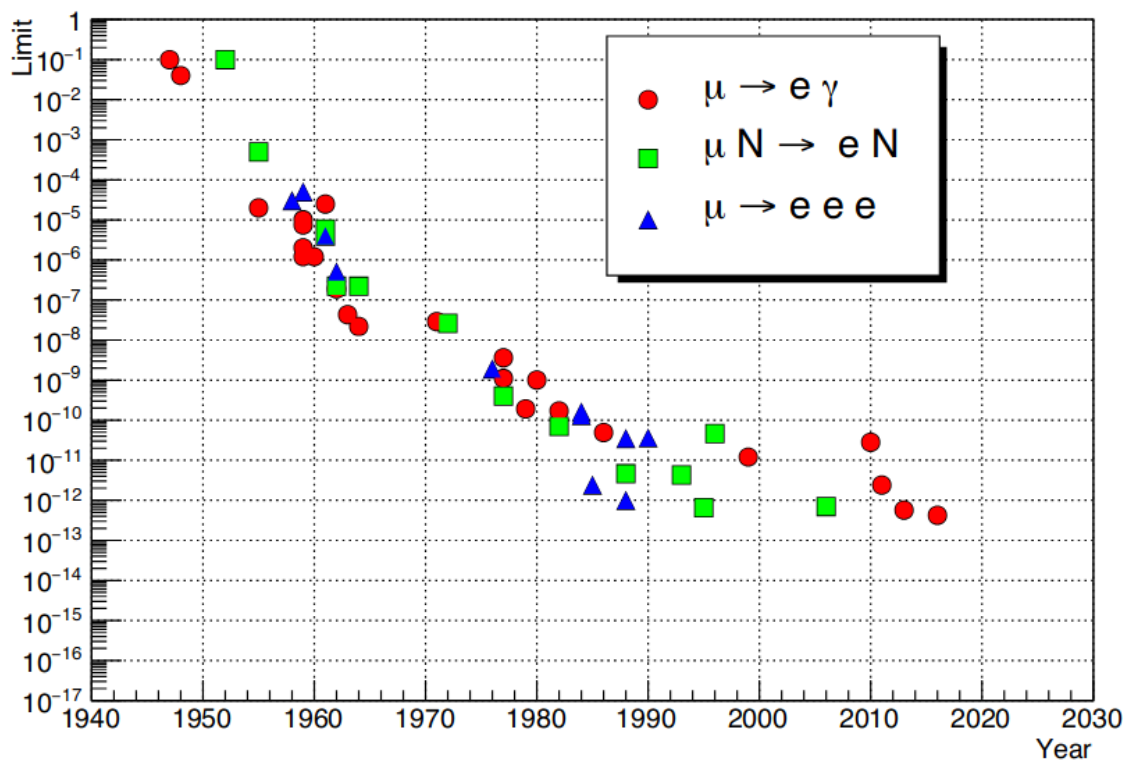

Figure 1.5: Limit on the branching ratio of flavor violation set by experiment as a function of the year [6]

The experiments that are currently under progress that aim to discover CLFV include:

- $M E G$ is an experiment where the lepton flavor violating muon decay mode $\mu^{+} \longrightarrow e^{+} \gamma$ was examined in the years 2009-2013 which resulted in establishing the best limit for the branching ratio so far at $4.2 \times 10^{-13}$. A $M E G$-II detector upgrade is under progress which aims to bring the sensitivity to $6 \times 10^{-14}$ [14] [15].

- DeeMee is an experiment at J-PARC MLF searching for Mu2e that aims at a single event sensitivity of $10^{-14}[16$.

- $М u 3 e$ is an experiment in search of lepton flavor violating muon decay mode $\mu^{+} \longrightarrow$ $e^{+} e^{-} e^{+}$that will be aiming to reach a sensitivity of $10^{-16}$ [17].

- The COMET experiment is searching for coherent neutrino-less $\mu^{-} \longrightarrow e^{-}$conversion in muonic atom of aluminum, $\mu^{-}+A l \longrightarrow e^{-}+A l$ at a sensitivity of $10^{-16}[18$. 
- Mu2e at Fermilab, which will be looking for Coherent neutrino-less $\mu^{-} \longrightarrow e^{-}$. We will be discussing this further in the following section.

\section{$1.4 M u 2 e$ at Fermilab [2][19]}

Fermilab houses many high intensity experiments and $M u 2 e$ is one of its prestigious undertakings. Fermilab's muon campus hosts two very ambitious experiments both of which are focused on the analysis of the same subatomic particle. Mu2e (Figure 1.7) is one, while $g-2$ [20] is the other. We will consider the requirements of the Mu2e experiment.

Mu2e at Fermilab aims to improve the current sensitivity limits of $\mu^{-} \longrightarrow e^{-}$by four orders of magnitude to $10^{-17}$. To achieve this sensitivity the experiment requires high intensity and a low energy muon beam. The negative muons from the beam are stopped in a target and captured by atoms. They undergo one of these three process:

- Decay in orbit $(\mathrm{DIO}) \Longrightarrow \mu^{-} \longrightarrow e^{-} \nu_{\mu} \overline{\nu_{e}}$ (predicted by the SM)

- Weak capture $\Longrightarrow \mu^{-} p \longrightarrow \nu_{\mu} n$ (predicted by the SM)

- Coherent flavor changing conversion $\Longrightarrow \mu^{-} N \longrightarrow e^{-} N$ [21] (proof of CLFV)

The coherent conversion is a powerful channel to search for CLFV, as it leaves the nucleus intact and there is only one detectable particle in the final state characterized by a signal consisting of mono-energetic electron with energy $E_{c e}$ :

$$
E_{c e}=m_{\mu}-E_{b}-\frac{E_{\mu}^{2}}{2 m_{N}}
$$

where $m_{\mu}$ is the muon mass at rest, $E_{b} \approx Z^{2} \alpha^{2} m_{\mu} / 2$ is the muonic atom binding energy for a nucleus with atomic number $Z, E_{\mu}$ is the nuclear recoil energy, and $m_{N}$ is the 
atomic mass. For this experiment we have a nucleus of aluminum which gives the value of $E_{c e}=104.963 \mathrm{MeV}$, which is a single peak of energy in an otherwise continous background spectrum. Background signals include DIO from high momentun tail of muons, pions and

electron-positron pair conversion of photon $(\gamma)$. Fermilab's accelerator complex (Figure 1.6) delivers the required high intensity muon beam to the target.

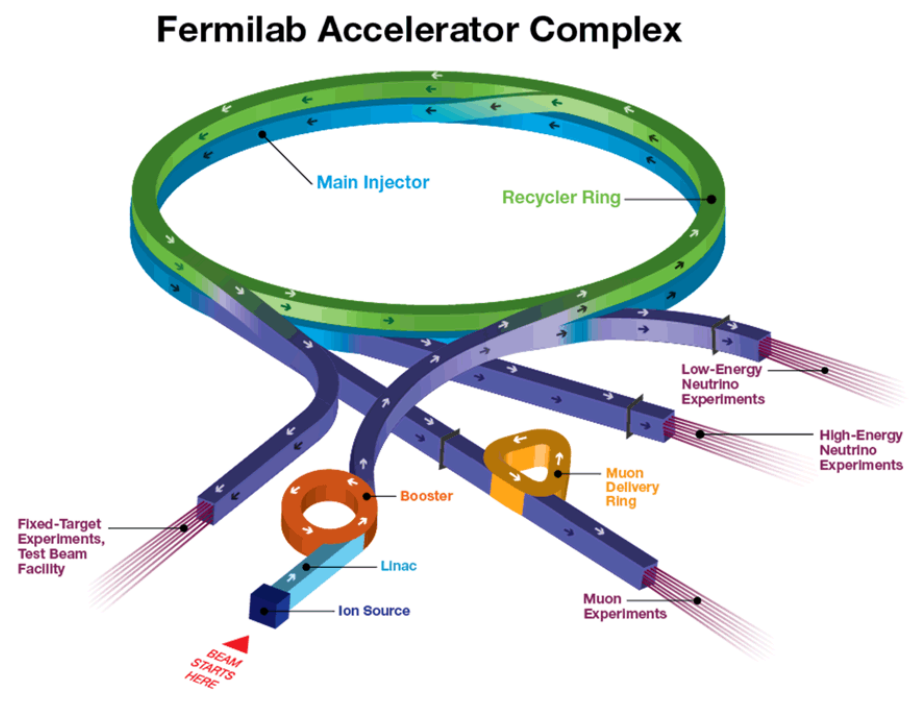

Figure 1.6: Fermilab Accelerator Campus [22]

The following section discusses how the necessary beam is transported for Mu2e.

\subsubsection{Beam Transport}

As the name of the experiment suggests, muons are the integral part of the experiment. Muons are formed from pion decays produced by a proton beam hitting a tungsten target, once the proton beam starts from the source it is accelerated through the Linac, Booster and Main Injector arriving in the Delivery Ring in bunches of $4 \times 10^{12}$ protons at $8 \mathrm{GeV}$. 
The full details of the acceleration are out of scope for this thesis and can be found in [2]. From the delivery ring, protons are extracted at a controlled rate towards extinction and to the target.

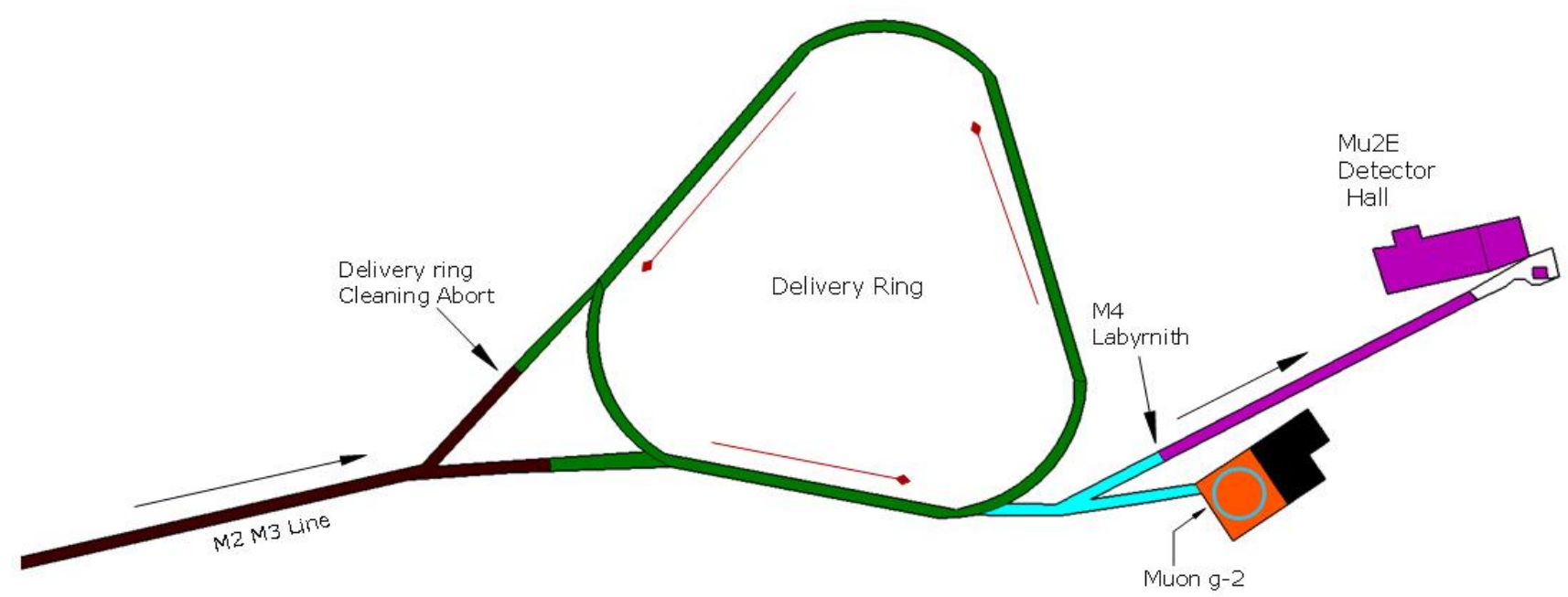

Figure 1.7: Muon Campus

\subsubsection{Extinction $[23]$}

Extinction is the process that comes directly after the extraction of the proton beam from the Delivery Ring. The Mu2e experiment requires a beam consisting of proton bunches 
$250 \mathrm{~ns}$ long, separated by $1.7 \mu \mathrm{s}$, with no out-of-time protons at the $10^{-10}$ fractional level to avoid backgrounds. The extinction process will make sure only the in-time beam is transmitted to the target. This is achieved by the AC dipole/collimator system. A bending dipole deflects the out-of-time beam such that it will be absorbed by a collimator downstream of the beam, thereby producing the required beam to the target.

\subsubsection{Target and Detector system [24]}

Once we get the proton beam with required intensity, the Target and Detector System generates and transports muons to the stopping target and detects the conversion to electrons. It consists of complex magnetic field configuration consisting of gradient fields, toroidal fields, and uniform fields generated with superconducting magnets. It is broadly divided into three sections: Production Solenoid, Transport Solenoid, and Detector Solenoid as shown in Figure 1.8 .

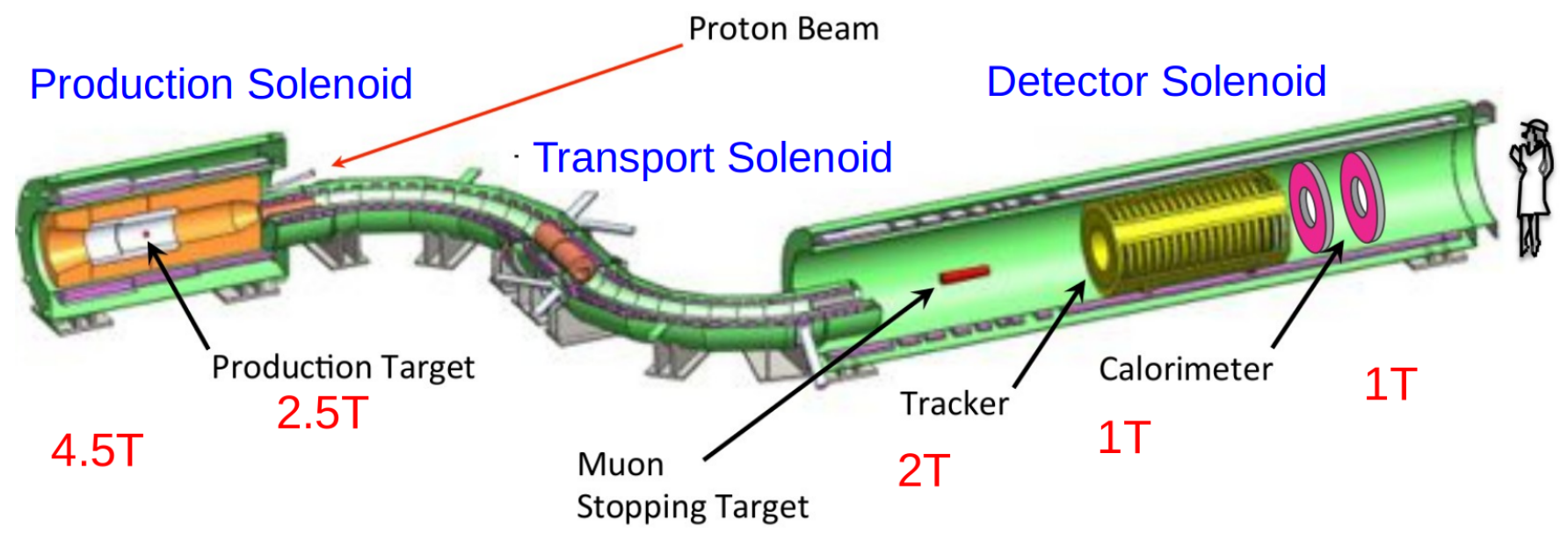

Figure 1.8: Production, Transport and Detector Solenoid systems [25] 
The first section includes the Production Solenoid where the $8 \mathrm{GeV}$ proton beam coming from right in Figure 1.8 will hit the tungsten target producing Kaons and charged Pions which will decay into muons that are transported away from initial beam direction to next section. This "backwards" mechanism is to avoid abundant unwanted particles like neutrons, photons, electrons or positrons from photon conversion and to absorb the remaining protons from the beam. This helps the detector capture events at a greater sensitivity. The Second section has the Transport Solenoid which transports the incoming muons from the first section towards the Detector Solenoid. It is designed with two bends and two collimators to filter high energy, negatively charged particles, positively charged particles, and neutral particles thereby reducing the background noise at the detector. The third and final section is the Detector Solenoid that has the stopping target (aluminum), the spectrometer, and the calorimeter for the detection of the conversion process of a muon into an electron.

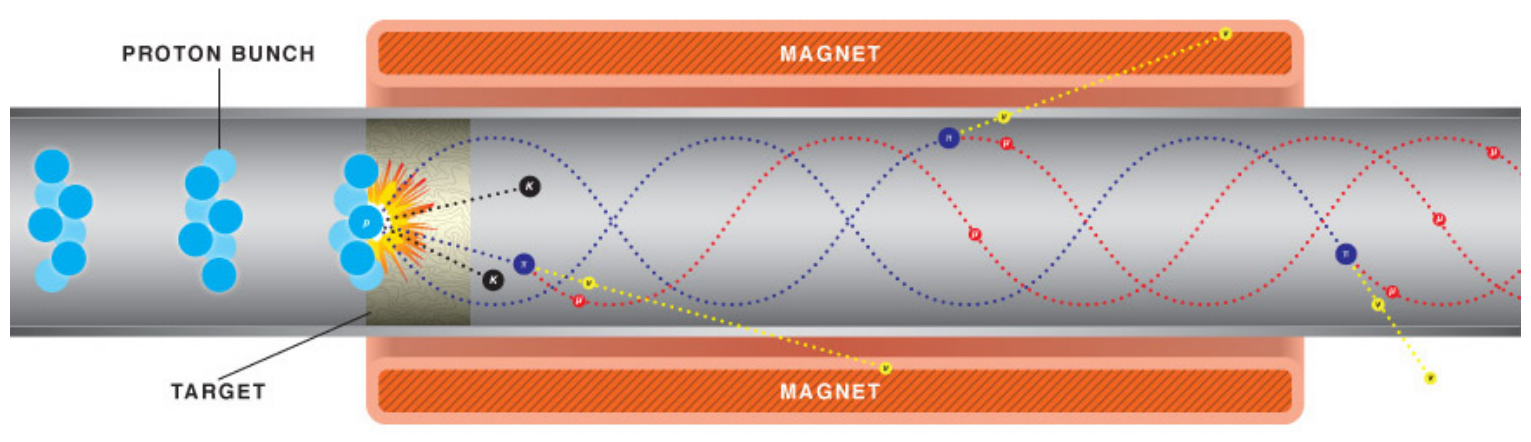

Figure 1.9: Production Target [25]

Because of the complexity involved in all these sections there are limitations to the number of protons that can be handled instantaneously at the Target and Detector system and this is the motivation for optimizing the design of the controlled extraction at the Delivery Ring. Slow Resonant Extraction is used to achieve this and is the main focus 
of this thesis. Before we discuss the extraction process, an understanding of the accelerator physics inside the Delivery Ring is required. 


\section{CHAPTER 2}

\section{ACCELERATOR PHYSICS}

This chapter will discuss the basics of accelerator physics, transverse dynamics, and

resonant extraction to understand how the high intensity proton beam will be delivered to the target for $M u 2 e$ in a controlled manner. The first half of the chapter will start by briefly discussing basic electromagnetism concepts, and then introduce a new coordinate system to use in accelerator calculations. The second half will use these concepts to discuss the essential beam dynamics necessary for understanding the main work in this thesis.

\section{$2.1 \quad$ Introduction}

Particle accelerators come in different shapes and sizes. Two typical geometry-based accelerators are circular and linear accelerators, which each have their own advantages and disadvantages [26]. Major factors that influence the design include:

- Particle to be transported (its type, mass, charge, etc..).

- Desired maximum energy to which particle is accelerated.

- Cost to build and operate the machine.

- Geographical location of the accelerator - largest accelerator built so far spans over an area with a circumference of $27 \mathrm{~km}$ so these machines can be huge. 
Accelerators use electromagnetic force to accelerate, steer and transport a particle beam from its source towards a target. Understanding how the particles interact with electromagnetism is important to understand the fundamentals of how accelerators work.

\subsection{Charged Particle in a Electromagnetic Field}

The classical theory of electromagnetism deals with electromagnetic fields and their interaction with charged particles. "Classical" implies the assumption that the charge distributions (sources) are localized and smooth. The macroscopic Maxwell's equations are stated as follows [27]:

$$
\begin{gathered}
\nabla \cdot \vec{D}=\rho_{\text {free }} \\
\nabla \cdot \vec{B}=0 \\
\nabla \times \vec{E}+\frac{\partial \vec{B}}{\partial t}=0 \\
\nabla \times \vec{H}-\frac{\partial \vec{D}}{\partial t}=j_{f r e e}
\end{gathered}
$$

Since the particles are traveling close to the speed of light, the Special Theory of Relativity also needs consideration. The energy ( $E$, different from electric field $\vec{E}$ ) of the particle with a momentum $\vec{P}$ and mass $m$ is given as,

$$
E=\sqrt{\vec{P}^{2} c^{2}+\left(m c^{2}\right)^{2}}
$$


Accelerator physicists use the kinetic energy $\left(E-m c^{2}\right)$ to describe the particle as it is the changing component in total energy. The force acting on a charged particle in an EM field is given by

$$
\vec{F}=\frac{d \vec{P}}{d t}=q(\vec{E}+\vec{v} \times \vec{B})
$$

where $\vec{E}$ and $\vec{B}$ are electric and magnetic fields respectively. The Hamiltonian of a charged particle in an electromagnetic field is given by

$$
H=\sqrt{\left(m c^{2}\right)^{2}+(\vec{P}-e \vec{A})^{2} c^{2}}+e \Phi
$$

where $\vec{A}$ is the vector potential and $\Phi$ is the scalar potential. Now that we have the basic tools from electromagnetism (EM), lets have a look at the coordinates that we use in accelerator systems.

\subsection{Coordinates in accelerator systems}

Conventional coordinates for the accelerator systems will make analysis of particle dynamics cumbersome. Redefining the coordinate system to better represent the particle trajectory in an accelerator allows for more ease when solving the equations for particle dynamics. Let's look at a general circular accelerator to see how the new coordinates work.

As shown in Figure 2.1, the ideal trajectory of a particle along the accelerator is considered as the origin of a locally inertial frame of reference moving along the path. Its transverse axes, $x$ and $y$ are perpendicular to the path, while the longitudinal axis $s$ is along the path length which is given by [28],

$$
\text { Path length }=\sqrt{g_{\mu \nu} d x^{\mu} d x^{\nu}}
$$




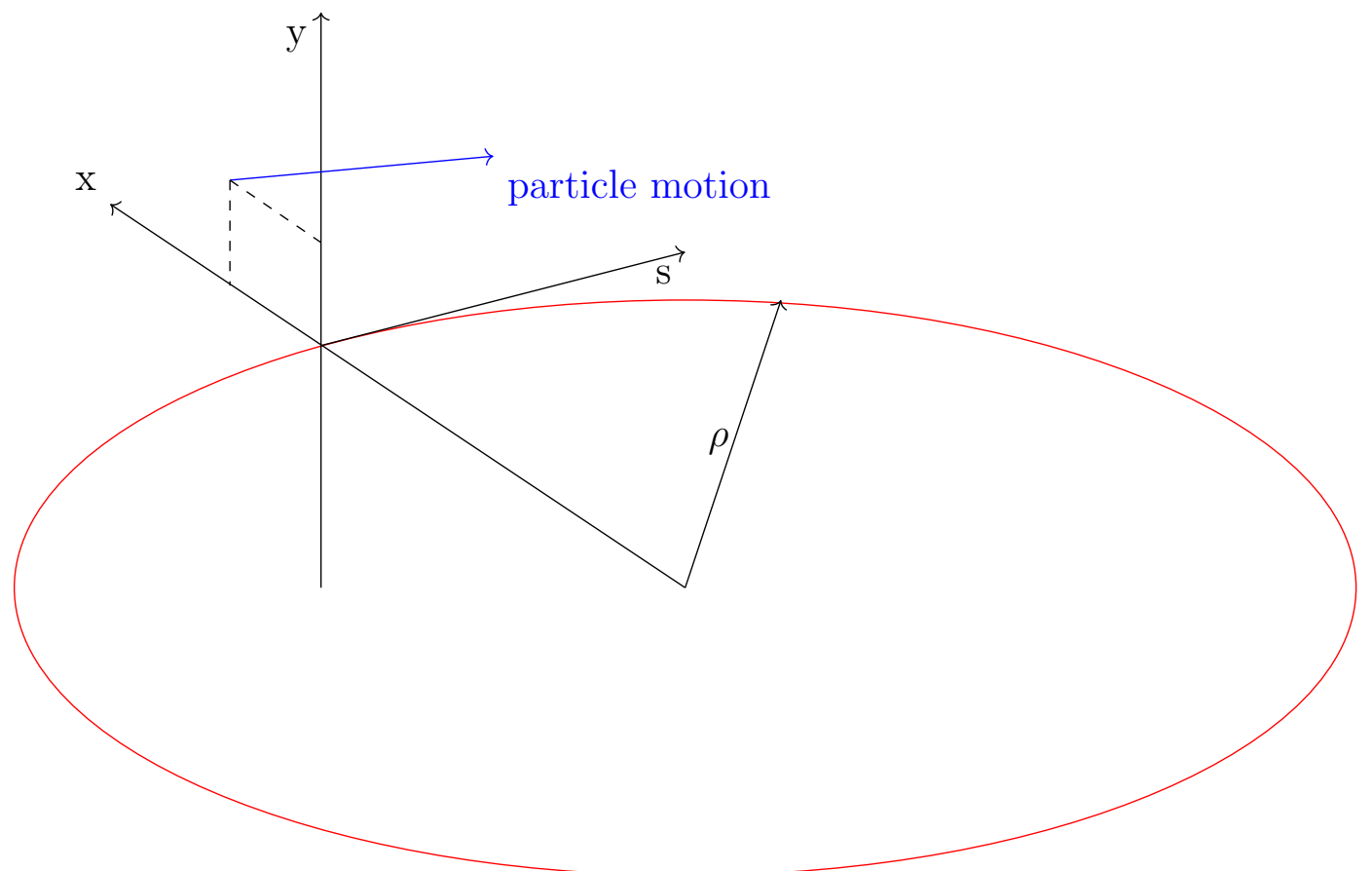

Figure 2.1: Coordinates in Particle Accelerators

Let us assume $\vec{r}_{o}(s)$ is the reference orbit of a particle and $\rho$ as the local radius of curvature, then the unit vectors for the coordinate system are as follows,

$$
\hat{s}(s)=\frac{d \vec{r}_{o}(s)}{d s}, \quad \hat{x}(s)=-\rho \frac{d \hat{s}(s)}{d s}, \quad \hat{y}(s)=\hat{x}(s) \times \hat{s}(s) .
$$

The differentials are then defined as follows:

$$
\frac{d \hat{s}(s)}{d s}=-\frac{1}{\rho} \hat{x}(s), \quad \frac{d \hat{x}(s)}{d s}=\frac{1}{\rho} \hat{s}(s)
$$

Assuming a reference orbit in planar geometry we have,

$$
\frac{d \hat{y}(s)}{d s}=0
$$


The position of the particle is then given by,

$$
\begin{gathered}
\vec{r}=\vec{r}_{o}(s)+x \hat{x}(s)+y \hat{y}(s) \\
\frac{d \vec{r}}{d s}=\hat{s}\left(1+\frac{x}{\rho}\right), \quad \frac{d \vec{r}}{d x}=\hat{x}, \quad \frac{d \vec{r}}{d y}=\hat{y} .
\end{gathered}
$$

Now that we know how to represent the particle in an accelerator system, we can rewrite the EM equations in the new coordinate system.

\subsubsection{Revisiting charged particle in an Electromagnetic Field}

Using these new coordinates the Hamiltonian (Eq 2.7) becomes

$$
H=c \sqrt{(m c)^{2}+\left(P_{x}-e A_{x}\right)^{2}+\left(P_{y}-e A_{y}\right)^{2}+\frac{1}{(1+x / \rho)^{2}}\left(P_{s}-e A_{s}\right)^{2}}+e \Phi
$$

Rearranging the above equation in terms of the new canonical variables, $\left[x, P_{x}\right],\left[y, P_{y}\right]$, and $[t,-H]$ gives the new Hamiltonian, $\mathscr{H}=-P_{s}$ :

$$
\mathscr{H}=-\sqrt{\left[\left(\frac{H-e \Phi}{c}\right)^{2}-(m c)^{2}-\left(P_{x}-e A_{x}\right)^{2}-\left(P_{y}-e A_{y}\right)^{2}\right]\left(1+\frac{x}{\rho}\right)^{2}}-e A_{s}
$$

A detailed derivation of equations 2.14 and 2.15 are given in Appendix A. A few assumptions that are applied for our case to help simplify the equations are as follows

- 2D magnetic field, $\vec{B}=B_{x} \hat{x}+B_{y} \hat{y}$, which leads to a vector potential with just $\mathrm{z}$ component and $A_{x}=A_{y}=0$

- Current study only includes magnetic elements, so there is no electric field which means $(H-e \Phi)$ can be written just as $\mathrm{H}$ 
- Constant Energy (no acceleration) which means

$$
\left(\frac{H}{c}\right)^{2}-(m c)^{2}=\vec{P}^{2}
$$

With these assumptions, the Hamiltonian becomes

$$
\mathscr{H}=-\sqrt{\left(\vec{P}^{2}-P_{x}^{2}-P_{y}^{2}\right)\left(1+\frac{x}{\rho}\right)^{2}}-e A_{s}
$$

Let $P_{0}$ be the momentum of the particle on reference orbit and $\vec{P}=P_{0}(1+\delta)$

$$
\tilde{\mathscr{H}}=\frac{\mathscr{H}}{P_{0}}=-\sqrt{\left(\frac{\vec{P}^{2}}{P_{0}^{2}}-\frac{P_{x}^{2}}{P_{0}^{2}}-\frac{P_{y}^{2}}{P_{0}^{2}}\right)\left(1+\frac{x}{\rho}\right)^{2}}-e \frac{A_{s}}{P_{0}}
$$

Let $\frac{P_{x}}{P_{0}}=\tilde{P}_{x}$ and $\frac{P_{y}}{P_{0}}=\tilde{P}_{y}$ then we have

$$
\begin{gathered}
\tilde{\mathscr{H}}=-\left(1+\frac{x}{\rho}\right) \sqrt{\left((1+\delta)^{2}-\tilde{P}_{x}^{2}-\tilde{P}_{y}^{2}\right)}-e \frac{A_{s}}{P_{0}} \\
\tilde{\mathscr{H}}=-\left(1+\frac{x}{\rho}\right)(1+\delta) \sqrt{\left(1-\frac{\tilde{P}_{x}^{2}}{(1+\delta)^{2}}-\frac{\tilde{P}_{y}^{2}}{(1+\delta)^{2}}\right)}-e \frac{A_{s}}{P_{0}}
\end{gathered}
$$

The following assumptions will further simplify the Hamiltonian

- Paraxial approximation $\tilde{P}_{x}, \tilde{P}_{y}<<1$

- Low momentum spread $\delta<<1$

Such that the Hamiltonian for a reference particle becomes:

$$
\tilde{\mathscr{H}}=-\left(1+\frac{x}{\rho}-\frac{x^{\prime 2}}{2}-\frac{y^{\prime 2}}{2}\right)-\frac{e}{P_{0}} \vec{A} \cdot \hat{s}\left(1+\frac{x}{\rho}\right)
$$


where,

$$
\begin{aligned}
x^{\prime} & =\frac{\partial \tilde{\mathscr{H}}}{\partial P_{x}}=\frac{\tilde{P}_{x}}{1+\delta} \\
y^{\prime} & =\frac{\partial \tilde{\mathscr{H}}}{\partial P_{y}}=\frac{\tilde{P}_{y}}{1+\delta}
\end{aligned}
$$

These new coordinates and canonical variables will be used from now on, which means we will be integrating over the path $s$ instead of time. A detailed analysis of how to arrive upon equation 2.21 can be found in Appendix A. The following section discusses the elements that will generate these fields inside the accelerator.

\subsection{Magnets}

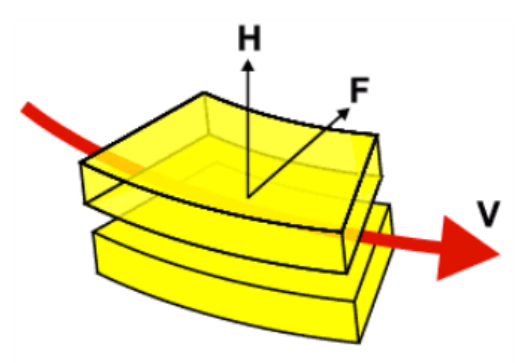

Figure 2.2: Dipole Magnet [29].

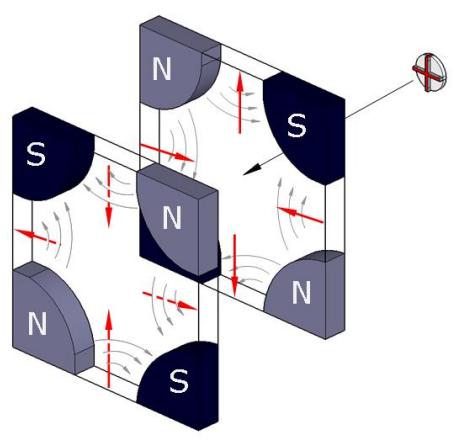

Figure 2.3: Quadrupole Magnets [30].

Magnets constitute major part of the accelerator that help us steer particles. In this section we will discuss in more detail about few common magnets that are in use.

A Dipole magnet (Figure 2.2) is used to steer particles only in one direction either vertically or horizontally. A Quadrupole will focus the particles in one direction and defocus 
in the other depending upon the poles placement as shown in Figure 2.3. A Sextupole is a magnet with non-linear field gradient i.e it will have higher focusing effect for the particles that are farther from center of the beam. These magnets are placed one behind another with a periodic arrangement of cells each of which contains focusing, defocusing, bending and drift elements. A simple such cell is called a FODO lattice. A particle accelerator contains many such lattice elements over its length.

Magnetic steering of particles is described mathematically as follows. From the assumptions in section 2.3.1 the magnetic field of any element in an accelerator is represented as $\vec{B}=B_{x} \hat{x}+B_{y} \hat{y}$ which results in

$$
\begin{gathered}
\nabla \times \vec{B}=\left|\begin{array}{ccc}
\hat{x} & \hat{y} & \hat{z} \\
\frac{\partial}{\partial x} & \frac{\partial}{\partial y} & 0 \\
B_{x} & B_{y} & 0
\end{array}\right|=\hat{z}\left(\frac{\partial B_{y}}{\partial x}-\frac{\partial B_{x}}{\partial y}\right)=0 \\
\Longrightarrow \frac{\partial B_{y}}{\partial x}=\frac{\partial B_{x}}{\partial y} \equiv B^{\prime}
\end{gathered}
$$

The taylor series expansion for magnetic field about $x=y=0$ for small $\mathrm{x}, \mathrm{y}$ gives:

$$
\vec{B} \approx\left(B_{x}(0,0)+\frac{\partial B_{x}}{\partial y} y+\frac{\partial B_{x}}{\partial x} x\right) \hat{x}+\left(B_{y}(0,0)+\frac{\partial B_{y}}{\partial x} x+\frac{\partial B_{y}}{\partial y} y\right) \hat{y}
$$

The Lorentz force in Eq2.6 can be rewritten as follows

$$
\frac{d \vec{P}}{d t}=q \vec{v} \times \vec{B}
$$




\subsubsection{Dipole}

For a dipole magnet with a non-zero magnetic field in y direction $\left(B_{y} \neq 0, B^{\prime}=0\right)$, the Lorentz force equation for a particle in position $\vec{R}=r \hat{x}+y \hat{y}$, where $r \equiv \rho+x$, gets simplified to

$$
\begin{gathered}
\gamma m \ddot{\vec{R}}=q\left(\vec{v} \times B_{y} \hat{y}\right) \\
\ddot{\vec{R}}=\frac{q v B_{y}}{\gamma m}=\frac{q v^{2} B_{y}}{\gamma m v}=\frac{q v^{2} B_{y}}{\vec{P}}=\frac{v^{2}}{\rho}
\end{gathered}
$$

which gives us the following relation for the bend radius $\rho$

$$
\frac{1}{\rho}=\frac{B_{y}}{(B \rho)}
$$

where $(B \rho)$ is called magnetic rigidity [26] and is given as;

$$
(B \rho) \equiv \frac{\vec{P}}{q}=\frac{\text { momentum }}{\text { charge }}
$$

\subsubsection{Quadrupole}

For a focusing quadrupole $B^{\prime} \neq 0$. Solving the Lorentz force equation for the quadrupole gives

$$
\vec{\gamma} \times \vec{B}=\left|\begin{array}{ccc}
\hat{x} & \hat{y} & \hat{\vec{s}}=q(\vec{v} \times \vec{B}) \\
v_{x} & v_{y} & v_{s} \\
B^{\prime} x & B^{\prime} y & 0
\end{array}\right|=-v_{s} B^{\prime} y \hat{x}+v_{s} B^{\prime} x \hat{y}+\left(v_{x} B^{\prime} x-v_{y} B^{\prime} y\right) \hat{s}
$$


Under the paraxial approximation we can ignore the third term in the above equation and treating $s$ as an independent variable, we have

$$
\frac{d}{d t}=\frac{d s}{d t} \frac{d}{d s}
$$

The above relations lead to two set of equations, defined as;

$$
\begin{gathered}
\gamma m v \frac{d^{2} x}{d s^{2}}=-q B^{\prime} x \\
\gamma m v \frac{d^{2} y}{d s^{2}}=q B^{\prime} y
\end{gathered}
$$

The definition in Eq2.30 gives the equations of motion are as follows

$$
\begin{aligned}
& \frac{d^{2} x}{d s^{2}}+\frac{B^{\prime}}{(B \rho)} x=0 \\
& \frac{d^{2} y}{d s^{2}}-\frac{B^{\prime}}{(B \rho)} y=0
\end{aligned}
$$

Both these equations have the form of a simple harmonic oscillator shown here:

$$
x^{\prime \prime}+K x=0(\text { Hills equation [26]) }
$$

For circular accelerators, $\mathrm{K}$ is periodic which gives the general solution for the above equation:

$$
x=A w(s) \cos [\psi(s)+\delta]
$$

where A, $\delta$ are two constants of integration. 


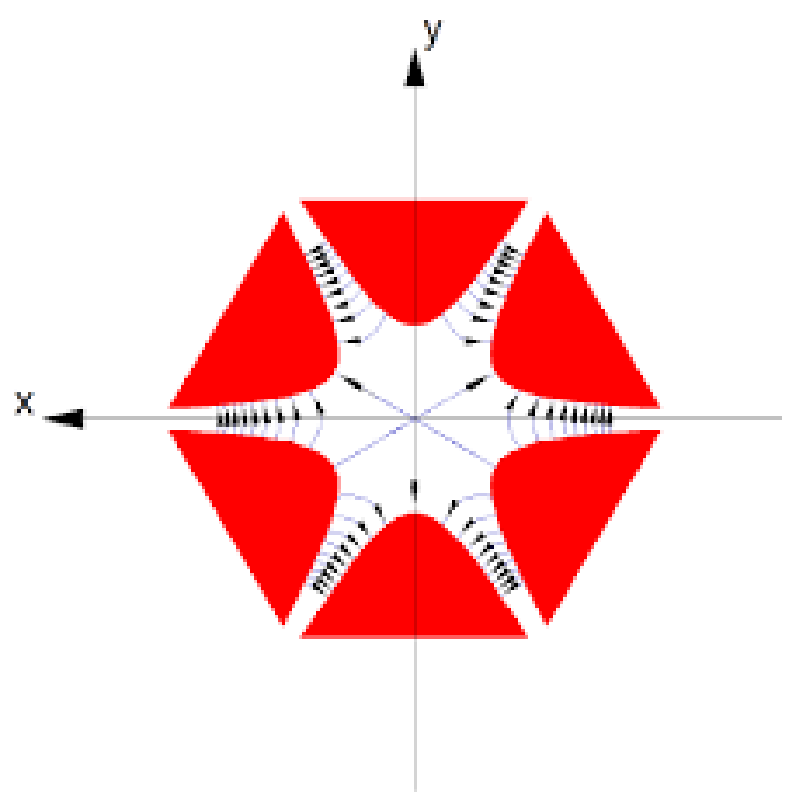

Figure 2.4: Sextupole 31]

\subsubsection{Sextupole}

A sextupole magnet has a gradient field which varies non-linearly with respect to position and is shown in Figure 2.4. Sextupoles are mainly used to correct the momentum spread of the beam (also known as chromaticity). The 2-D field components within a sextupole magnet are

$$
\begin{gathered}
B_{x}=B^{\prime \prime} x y \\
B_{y}=\frac{B^{\prime \prime}\left(x^{2}-y^{2}\right)}{2}
\end{gathered}
$$

where $B^{\prime \prime}$ is a constant, and so following the same procedure as for quadrupoles, we have:

$$
\vec{v} \times \vec{B}=\left|\begin{array}{ccc}
\hat{x} & \hat{y} & \hat{s} \\
v_{x} & v_{y} & v_{s} \\
B^{\prime \prime} x y & \frac{B^{\prime \prime}\left(x^{2}-y^{2}\right)}{2} & 0
\end{array}\right|=-v_{s} \frac{B^{\prime \prime}}{2}\left(x^{2}-y^{2}\right) \hat{x}+v_{s} B^{\prime \prime} x y \hat{y}
$$


And the equations of motion (ignoring the coupled motion) are as follows

$$
\begin{aligned}
& \frac{d^{2} x}{d s^{2}}+\frac{B^{\prime \prime}}{(2 B \rho)} x^{2}=0 \\
& \frac{d^{2} y}{d s^{2}}-\frac{B^{\prime \prime}}{(2 B \rho)} y^{2}=0
\end{aligned}
$$

Many accelerators contain a combination of all three magnets discussed so far to control the transverse motion. There are new accelerators being built with additional non-linear elements like octupole, decapoles; However that discussion is out of the scope of this thesis.

\subsection{Courant-Snyder Parameters [32]}

In region of constant $K$, The equation of motion (Eq 2.37) can also be expressed in the matrix form [26] as follows,

$$
\left(\begin{array}{c}
x \\
x^{\prime}
\end{array}\right)_{\text {out }}=\left(\begin{array}{cc}
\cos (\sqrt{K} l) & \frac{1}{\sqrt{K}} \sin (\sqrt{K} l) \\
-\sqrt{K} \sin (\sqrt{K} l) & \cos (\sqrt{K} l)
\end{array}\right)\left(\begin{array}{c}
x \\
x^{\prime}
\end{array}\right)_{s_{0}}
$$

If $K<0$, then

$$
\left(\begin{array}{c}
x \\
x^{\prime}
\end{array}\right)_{\text {out }}=\left(\begin{array}{cc}
\cosh (\sqrt{|K|} l) & \frac{1}{\sqrt{|K|}} \sinh (\sqrt{|K|} l) \\
-\sqrt{|K|} \sinh (\sqrt{|K|} l) & \cosh (\sqrt{|K|} l)
\end{array}\right)\left(\begin{array}{c}
x \\
x^{\prime}
\end{array}\right)_{s_{0}}
$$

Using the general solution from Eq 2.38 we can write the equation of motion as follows,

$$
\left(\begin{array}{c}
x \\
x^{\prime}
\end{array}\right)_{s_{0}+C}=\left(\begin{array}{cc}
\cos \Delta \psi_{C}+\alpha \sin \Delta \psi_{C} & \beta \sin \Delta \psi_{C} \\
-\gamma \sin \Delta \psi_{C} & \cos \Delta \psi_{C}-\alpha \sin \Delta \psi_{C}
\end{array}\right)\left(\begin{array}{c}
x \\
x^{\prime}
\end{array}\right)_{s_{0}}
$$


where $\mathrm{C}$ is distance along a periodic section and,

$$
\begin{gathered}
\beta(s) \equiv \frac{w^{2}(s)}{k}, \psi^{\prime}=\frac{k}{w(s)^{2}} \\
\alpha(s) \equiv-\frac{1}{2} \frac{d \beta(s)}{d s} \\
\gamma \equiv \frac{1+\alpha^{2}}{\beta}
\end{gathered}
$$

These quantities $\alpha, \beta$ and $\gamma$ are called the Courant-Snyder parameters. A detailed derivation of the above matrix solution is given in Appendix B. The Phase advance of the particle for one turn is given by:

$$
\Delta \psi_{C}=\int_{s_{0}}^{s_{0}+C} \frac{d s}{\beta(s)}
$$

The beta function will determine how the particles evolve around the accelerator based on their initial position. The number of particle oscillations about the reference orbit per turn is called tune of the accelerator and is an important parameter characterizing accelerator lattice. Tune can be calculated as:

$$
\nu=\frac{1}{2 \pi} \oint \frac{d s}{\beta(s)}
$$

\subsubsection{Ellipses}

All the discussion so far has described a single particle's trajectory. However an accelerator is never built to transport a single particle, and usually has a particle count to the order of $10^{12}$ or higher. So, the formalism of the dynamics will be extended for an ensemble of 


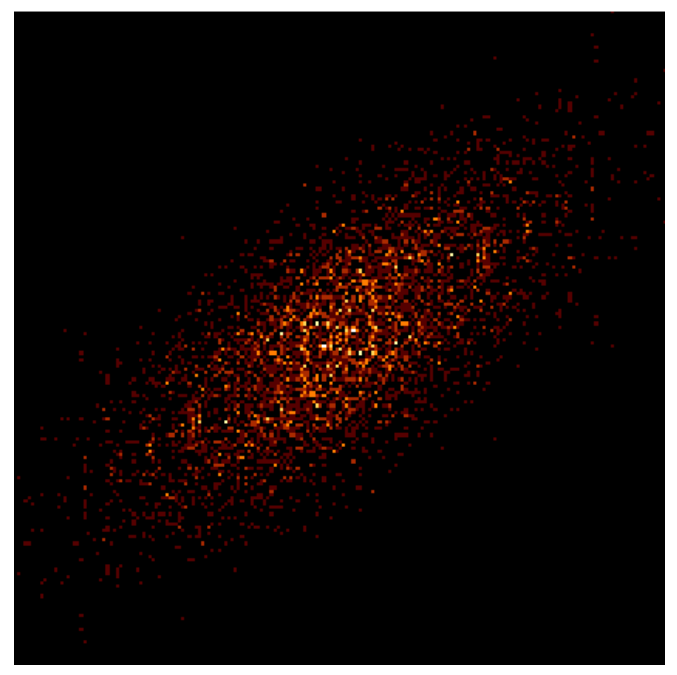

Figure 2.5: Particle distribution.

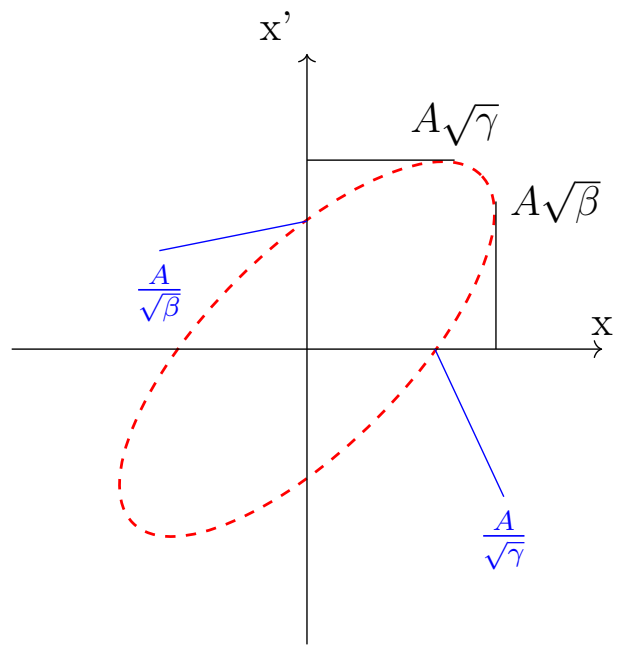

Figure 2.6: Phase space mapping.

particles. The solution for the equations of motion in terms of Courant-Synder parameters is 33 ,

$$
x=A \sqrt{\beta(s)} \cos [\psi(s)+\delta]
$$

Rearranging the equation and expressing $\mathrm{A}$ in terms of $x$ and $x^{\prime}$ gives

$$
A^{2}=\gamma x^{2}+2 \alpha x x^{\prime}+\beta x^{\prime 2}
$$

which is similar to the general equation of an ellipse. It follows that the phase space area of particles traveling around the circular accelerator looks as shown in Figures 2.5 and 2.6. The phase space area occupied by the beam is called emittance $(\epsilon)$ which has units of mm-mrad or sometimes $\pi$ mm-mrad. Includng emittance, Eq 2.53 can be rewritten as follows

$$
\frac{\epsilon}{\pi}=\gamma x^{2}+2 \alpha x x^{\prime}+\beta x^{\prime 2}
$$


Emittance depends on the energy of the beam, normalized emittance is used to describe the phase space area independent of the beam energy as follows,

$$
\epsilon_{n}=\epsilon \times(\gamma \beta)
$$

where $\gamma$ and $\beta$ are relativistic parameters. For an ensemble of particles, the Courant-Snyder parameters can also be given in terms of a statistical quantity like the standard deviation [34] $(\sigma)$ :

$$
\beta=\frac{\pi \sigma_{x}^{2}}{\epsilon} ; \quad \gamma=\frac{\pi \sigma_{x^{\prime}}^{2}}{\epsilon} ; \quad \alpha=-\frac{\pi \sigma_{x} \sigma_{x^{\prime}}}{\epsilon}
$$

\subsubsection{Normalized Phase Space}

The elliptical phase space can be transformed into normalized circular phase space (see Figures 2.7 and 2.8 by the transformation of coordinates as follows

$$
\begin{gathered}
x=a\left(\frac{\beta(s)}{\beta_{0}}\right)^{1 / 2} \cos \chi(s) \\
p_{x} \equiv \beta(s) x^{\prime}+\alpha(s) x=-a\left(\frac{\beta(s)}{\beta_{0}}\right)^{1 / 2} \sin \chi(s)
\end{gathered}
$$

where $a$ is the real amplitude at the location of interest where $\beta=\beta_{0}$. 


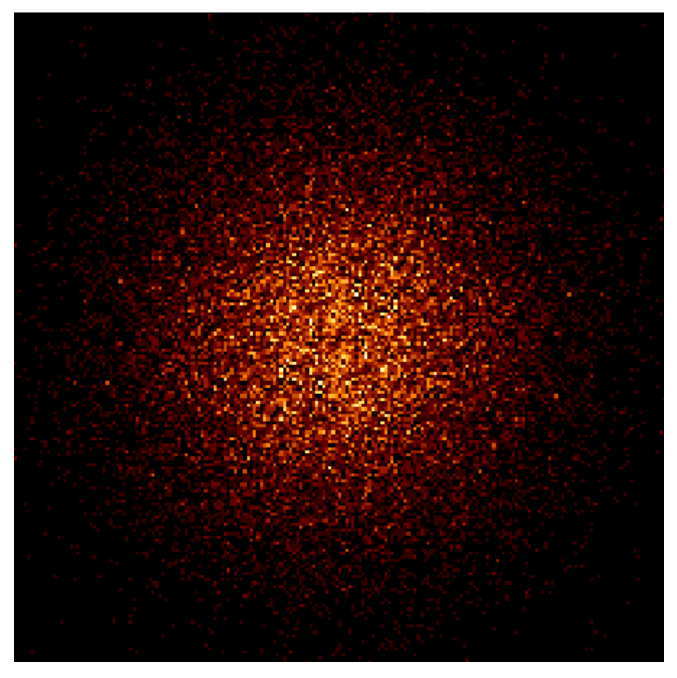

Figure 2.7: Normalized Particle distribution.

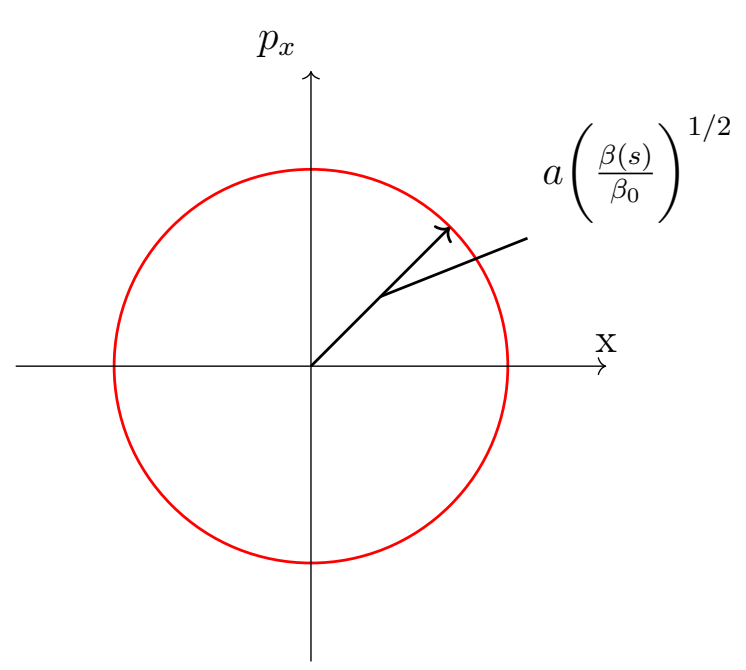

Figure 2.8: Normalized Phase Space.

\subsection{Resonance}

Whenever there is periodic motion there can be resonance conditions which might make the system unstable. This section will look at resonance condition in an accelerator and the effect it has on the particle beam. For clarification, the resonance being discussed here is in the transverse direction.

\subsubsection{Dipole Resonance}

Consider an accelerator with a dipole error at a single location due to either manufacturing defect or a construction error. If the tune of the machine is integer (say i) then a particle in phase space would ideally arrive at the same position after i turns. For example, if $\mathrm{i}=1$, the particle should arrive at the same spot at each turn. However dipole error will cause the particle to slowly get a kick outwards each turn. With a finite aperture, the particle will 


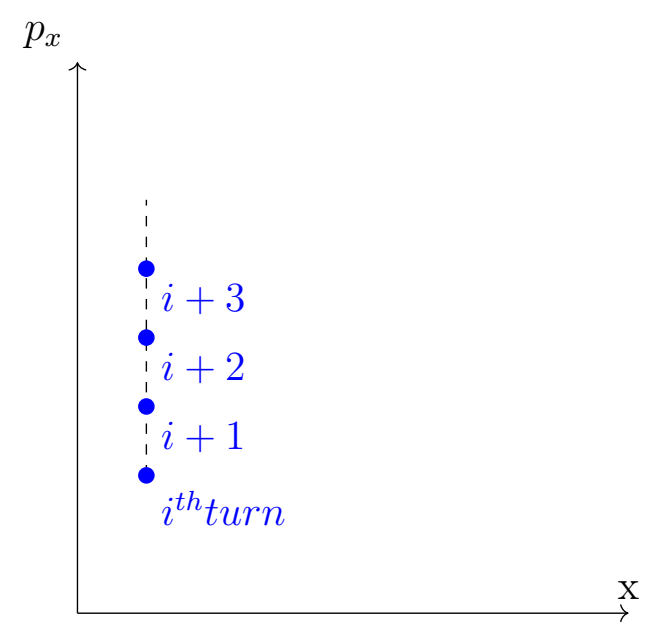

Figure 2.9: Dipole Resonance.

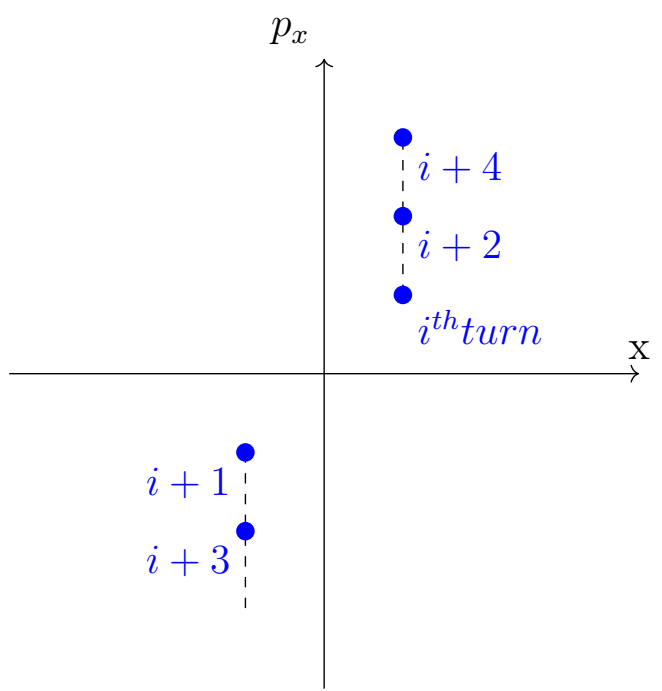

Figure 2.10: Quadrupole Resonance.

be lost. The particle's trajectory is represented in Figure 2.9 for a dipole error at a random location.

\subsubsection{Quadrupole and Sextupole Resonances}

Using similar qualitative argument, if we have a single quadrupole error and the machine is running at a half integer tune the particle would receive a kick every second turn as shown in Figure 2.10. For a sextupole field error, if the tune is close to one-third of an integer then the particle will receive a kick every third turn as shown in Figure 2.11. In this thesis we will focus on sextupole resonance which will be discussed more rigorously in following sections.

In all cases of resonance, the particle amplitude is increasing, so if we have an ensemble of particles the beam size increases at each turn. In the following section, we use the increasing beam size to our advantage. 


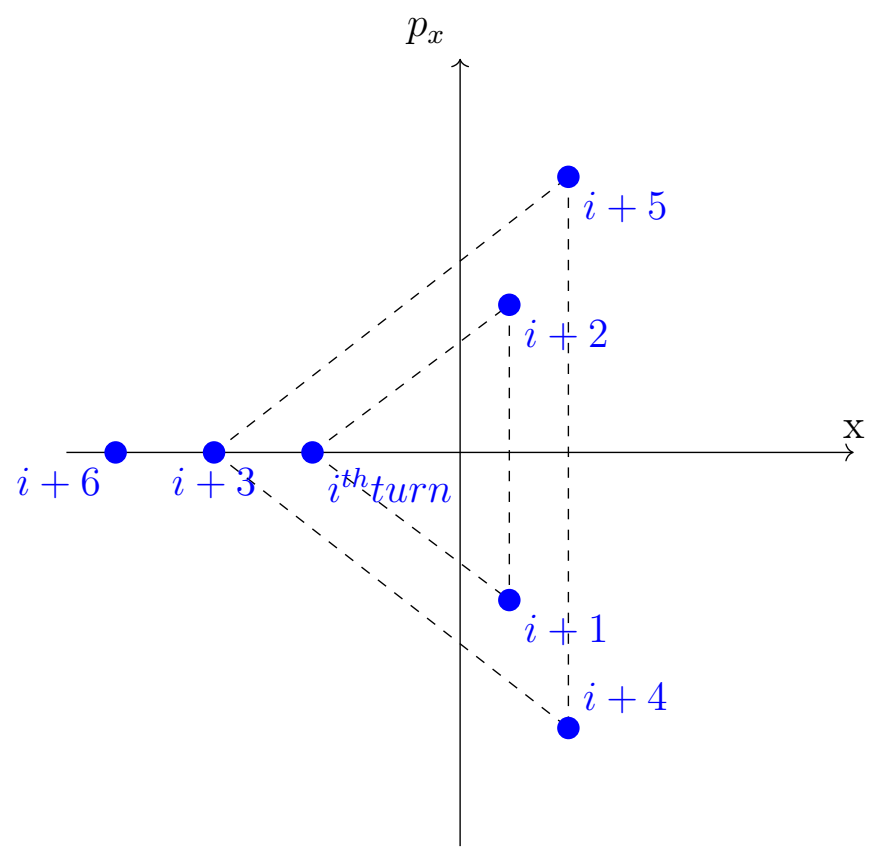

Figure 2.11: Sextupole Resonance.

\subsection{Resonant Extraction [26]}

Resonance extraction is a clever method of controlling and changing the phase space of particles by introducing a small perturbation in the field. Assume a small perturbation introduced in the field $\Delta B(x, s)$, particle is deflected by an angle and its slope changes as follows,

$$
\Delta x^{\prime}=-\frac{\Delta B \Delta s}{B \rho}
$$

As a result,

$$
\Delta p_{x}=-\beta(s) \frac{\Delta B \Delta s}{B \rho}
$$


The particle's amplitude and phase change due to the perturbation for infinitesimal $\Delta s$, can be followed from the equations

$$
\begin{gathered}
\Delta x=\left(\frac{\beta}{\beta_{0}}\right)^{1 / 2}(\Delta a \cos \chi-a \sin \chi \cdot \Delta \chi)=0 \\
\Delta p_{x}=-\left(\frac{\beta}{\beta_{0}}\right)^{1 / 2}(\Delta a \sin \chi+a \cos \chi \cdot \Delta \chi)=-\beta(s) \frac{\Delta B \Delta s}{B \rho}
\end{gathered}
$$

which results in,

$$
\begin{aligned}
& \Delta a=\frac{\beta_{0}}{(B \rho)}\left(\frac{\beta}{\beta_{0}}\right)^{1 / 2} \Delta B \Delta s \sin \chi \\
& \Delta \chi=\frac{\beta_{0}}{(B \rho)}\left(\frac{\beta}{\beta_{0}}\right)^{1 / 2} \frac{\Delta B \Delta s}{a} \cos \chi
\end{aligned}
$$

These perturbations add up for each turn and the phase advances as per

$$
\chi(s)=\psi+\nu \phi(s)
$$

where,

$$
\phi(s) \equiv \int \frac{d s}{\nu \beta(s)}
$$

As we add up all the effects of individual perturbations experienced by the particle along its path we have,

$$
\frac{d a}{d n}=\frac{\beta_{0}}{(B \rho)} \oint\left(\frac{\beta}{\beta_{0}}\right)^{1 / 2} \Delta B \sin (\psi+\nu \phi) d s .
$$

Change in the phase after passing through one turn is,

$$
\Delta \psi=2 \pi \nu+\frac{\beta_{0}}{(B \rho)} \oint\left(\frac{\beta}{\beta_{0}}\right)^{1 / 2} \frac{\Delta B}{a} \cos (\psi+\nu \phi) d s
$$


Assuming a difference between phase advance and $2 \pi \nu$ to be small, we have

$$
\frac{d}{d n}(\psi-2 \pi \nu)=\frac{\beta_{0}}{(B \rho)} \oint\left(\frac{\beta}{\beta_{0}}\right)^{1 / 2} \frac{\Delta B}{a} \cos (\psi+\nu \phi) d s .
$$

\subsubsection{Non-linear Resonance using Sextupoles}

Now that we have seen the effects of perturbations on the phase space of the particles let's see what happens if the perturbation comes from a sextupole magnet. For a sextupole magnet,

$$
\Delta B(x, s)=\frac{B^{\prime \prime}(s)}{2} x^{2}
$$

Substituting the above value into equations 2.67 and 2.68 gives

$$
\frac{d a}{d n}=a^{2} \frac{\beta_{0}}{(B \rho)} \oint\left(\frac{\beta}{\beta_{0}}\right)^{3 / 2} \frac{B^{\prime \prime}}{2} \cos ^{2} \chi \sin \chi(s) d s
$$

Using a few trigonometric identities we have

$$
\begin{gathered}
\frac{d a}{d n}=\frac{a^{2}}{4} \frac{\beta_{0}}{(B \rho)} \oint\left(\frac{\beta}{\beta_{0}}\right)^{3 / 2} \frac{B^{\prime \prime}}{2}(\sin \chi+\sin 3 \chi) d s \\
\frac{d a}{d n}=\frac{a^{2}}{4} \frac{\beta_{0}}{(B \rho)} \oint\left(\frac{\beta}{\beta_{0}}\right)^{3 / 2} \frac{B^{\prime \prime}}{2}[\sin \psi \cos \nu \phi+\cos \psi \sin \nu \phi \\
+\sin 3 \psi \cos 3 \nu \phi+\cos 3 \psi \sin 3 \nu \phi] d s
\end{gathered}
$$


If the tune $(\nu)$ is close to third integer $\left(\nu_{0}=m / 3, m=\right.$ integer $)$, the $\sin 3 \chi$ contribution for sextupoles dominates $\sin \chi$, so we have,

$$
\frac{d a}{d n}=\frac{a^{2}}{4} \frac{\beta_{0}}{(B \rho)} \oint\left(\frac{\beta}{\beta_{0}}\right)^{3 / 2} \frac{B^{\prime \prime}}{2}\left[\sin 3 \psi \cos 3 \nu_{0} \phi+\cos 3 \psi \sin 3 \nu_{0} \phi\right] d s
$$

Similarly,

$$
\frac{d \psi}{d n}=\frac{a}{4} \frac{\beta_{0}}{(B \rho)} \oint\left(\frac{\beta}{\beta_{0}}\right)^{3 / 2} \frac{B^{\prime \prime}}{2}\left[\cos 3 \psi \cos 3 \nu_{0} \phi-\sin 3 \psi \sin 3 \nu_{0} \phi\right] d s
$$

Replacing $\psi$ with $\tilde{\psi} \equiv \psi-2 \pi \nu_{0} n$ these equations can be written as

$$
\begin{gathered}
\frac{d a}{d n}=\frac{a^{2}}{4}(A \sin 3 \tilde{\psi}+B \cos 3 \tilde{\psi}) \\
\frac{d \tilde{\psi}}{d n}=\frac{a}{4}(A \cos 3 \tilde{\psi}-B \sin 3 \tilde{\psi})+2 \pi \delta
\end{gathered}
$$

where $\delta=\nu-\nu_{0}$ and,

$$
\begin{aligned}
A & \equiv \frac{\beta_{0}}{(B \rho)} \oint\left(\frac{\beta}{\beta_{0}}\right)^{3 / 2} \frac{B^{\prime \prime}}{2} \cos 3 \nu_{0} \phi d s \\
B & \equiv \frac{\beta_{0}}{(B \rho)} \oint\left(\frac{\beta}{\beta_{0}}\right)^{3 / 2} \frac{B^{\prime \prime}}{2} \sin 3 \nu_{0} \phi d s
\end{aligned}
$$

\subsubsection{Equations of motion for sextupole resonance}

By rotating back to our canonical variables $x, p_{x}$ we have the following equations of motion

$$
\begin{aligned}
\frac{d \tilde{x}}{d n} & =\frac{\tilde{x}}{a}\left(\frac{d a}{d n}\right)+\tilde{p_{x}}\left(\frac{d \tilde{\psi}}{d n}\right) \\
\frac{d \tilde{p_{x}}}{d n} & =\frac{\tilde{p_{x}}}{a}\left(\frac{d a}{d n}\right)-\tilde{x}\left(\frac{d \tilde{\psi}}{d n}\right),
\end{aligned}
$$


After careful algebra we get,

$$
\begin{aligned}
& \frac{d \tilde{x}}{d n}=\frac{1}{4} A\left(-2 \tilde{x} \tilde{p_{x}}\right)+\frac{1}{4} B\left(\tilde{x}^{2}-{\tilde{p_{x}}}^{2}\right)+2 \pi \delta \tilde{p_{x}} \\
& \frac{d \tilde{p_{x}}}{d n}=\frac{1}{4} A\left(\tilde{x}^{2}-{\tilde{p_{x}}}^{2}\right)+\frac{1}{4} B\left(-2 \tilde{x} \tilde{p_{x}}\right)-2 \pi \delta \tilde{x}
\end{aligned}
$$

consider a case where $\mathrm{B}=0$, then by solving the above set of equations we have,

$$
\left(\tilde{x}-\frac{4 \pi \delta}{A}\right)\left[\tilde{P}_{x}^{2}-\frac{1}{3}\left(\tilde{x}+\frac{8 \pi \delta}{A}\right)^{2}\right]=k(\text { constant })
$$

Fixed points in the separatrix (separatrix constitutes the boundaries for stable phase space region) are derived by solving the above equation for cases $d \tilde{x} / d n=0$ and $d \tilde{P}_{x} / d n=0$ which are

$$
\left(\tilde{x}=-\frac{8 \pi \delta}{A}, \tilde{P}_{x}=0\right)
$$

and

$$
\left(\tilde{x}=-\frac{4 \pi \delta}{A}, \tilde{P}_{x}= \pm \sqrt{3} \frac{4 \pi \delta}{A}\right),
$$

The separatrix is shown in the Figure 2.12. If there is more than one sextupole (i.e, for arbitrary values of $A$ and $B$ ), the orientation of the separatrix can be controlled as seen in Figure 2.13. The red line in the Figure 2.13 is the resultant orientation of the separatrix when two sextupoles are separated by a phase difference of $3 \Delta \psi_{0}=90^{\circ}$. Any particles outside of the separatrix will escape away from the bounded phase space over a number of turns.

The next chapter will use the analytical information discussed here in Chapter 2 to describe the experimental setup and simulate resonance extraction for $M u 2 e$. 


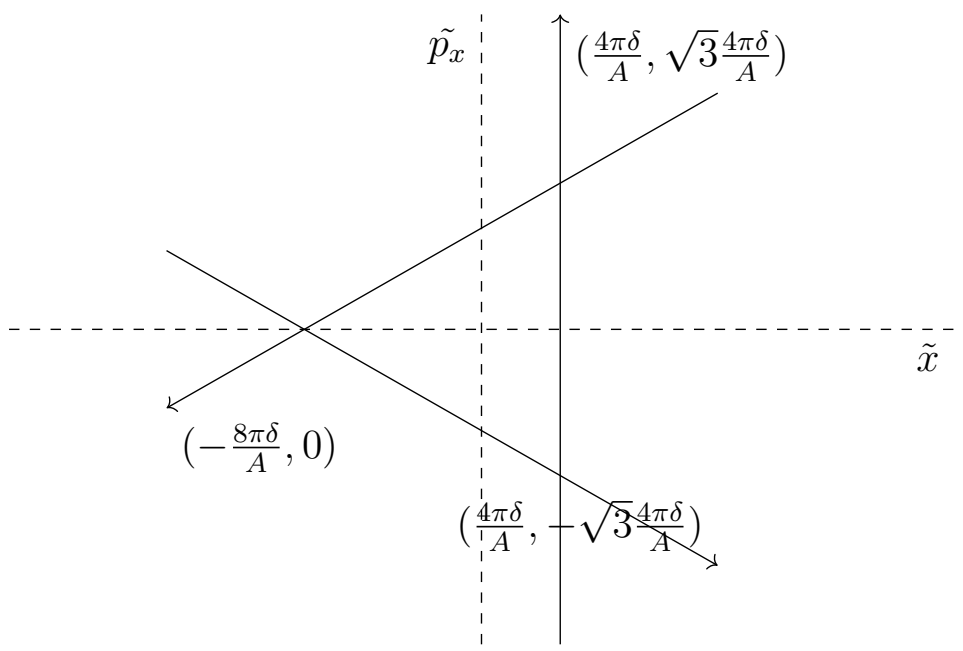

Figure 2.12: Separatrix for sextupole resonance

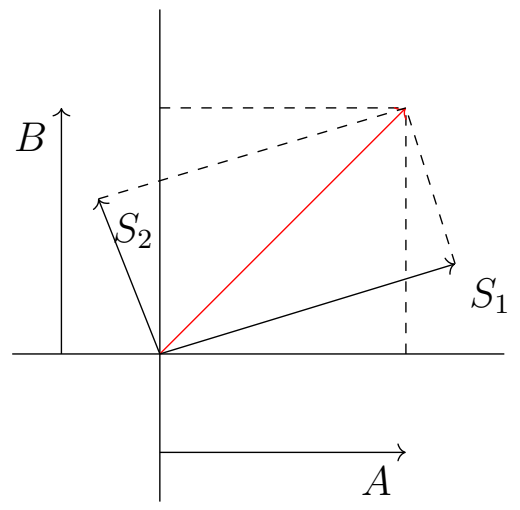

Figure 2.13: Two sextupoles 


\section{CHAPTER 3 SIMULATION}

Previous chapters discussed briefly about the motivations behind $M u 2 e$, and the details about the experimental setup at Fermilab. We also learned about the mathematical tools that help us understand accelerator physics necessary for $M u 2 e$, now let's look at the actual simulation to see whether we can extract beam towards the target at required rate to look for muon to electron conversion.

\subsection{Simulation}

In chapter 2, the Courant-Snyder (CS) parameters, and the equations of motion for a particle in circular accelerator were introduced. All these equations are helpful tracing

the particle's trajectory in the accelerator. To ease the burden of tracking the motion of thousands of particles simultaneously, we make use of two simulation softwares: R and MAD$\mathrm{X}$. The analysis of particle motion starts from the moment particle enters the Delivery Ring (DR) until it reaches the extraction point, focusing mainly on the transverse dynamics. The assumption remains that the particles don't experience any acceleration during this time. Even though the DR is not circular in shape, it is periodical and we can therefore use our equations of motion to track the particles each turn. 


\subsection{1 $\mathrm{R}$ and MAD-X}

$\mathrm{R}[35]$ is a powerful statistical programming language useful for analysis on large dataframes. In the case of $M u 2 e$, we can perform matrix operations on thousands of particles simultaneously using R. CS parameters and phase advance allows for use of the matrix representation to track particle through any element.

Methodical Accelerator Design (MAD[36]) is a software by CERN aimed at performing computations for particle accelerators. It is a powerful tool that can track a particle through any common element used in accelerators. MAD allows for definition of parameters for each element independently. For example a quadrupole magnet can be defined with its length, gradient, and tilt as parameters which allows for more flexibility. One can automate the process to get a range of parameters for a certain element to arrive at the desired output values. MAD can calculate the Twiss parameters, which include canonical variables at each element apart from tune, dispersion and other CS parameters. Current version of MAD is MAD-X.

\subsection{Setup and Parameters}

The Delivery Ring (DR) at Fermilab is $505 \mathrm{~km}$ long and contains about one thousand elements to be included in the MAD-X simulation. There is a mirror symmetry with a period of three in the lattice. The three way symmetry can be seen from the betatron oscillation plot as show in Figure 3.1 . 

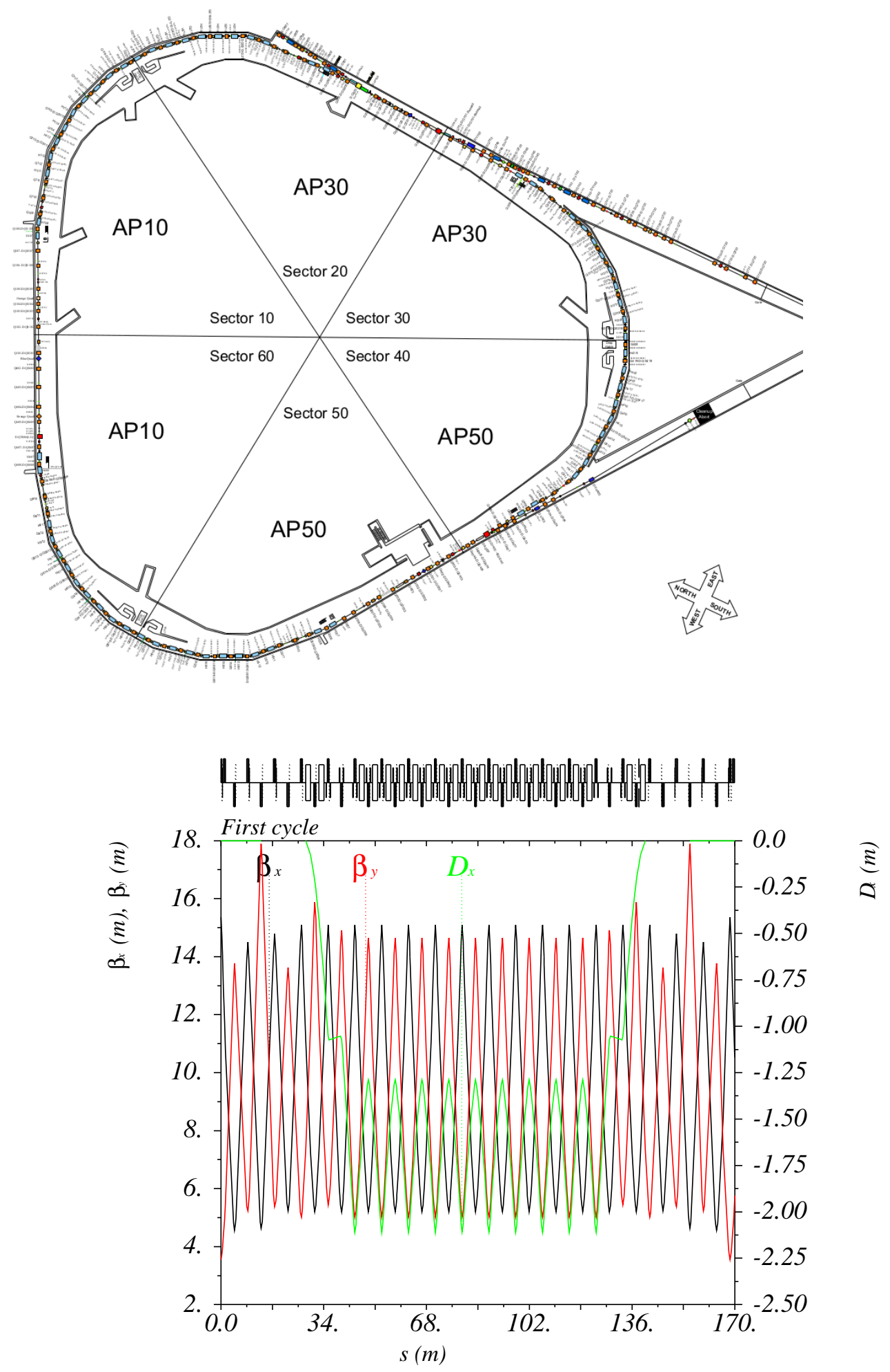

Figure 3.1: Delivery Ring (DR) Lattice[37] and amplitude function of $\beta_{x}$ and $\beta_{y}$ for each side of the DR 
The plot in Figure 3.1 includes dispersion values, which will be ignored in future simulations for the sake of simplicity. A few parameters used to setup the simulation in MAD-X are shown in Table 3.1. Some of these are constants from the designed parameters of the Mu2e experiment, while the others are an approximation assumed for the ease of simulation.

Table 3.1: Parameters that are considered for this simulation [2].

\begin{tabular}{|c||c|}
\hline Type & value \\
\hline \hline Emittance(normalized) & $16 \pi \mathrm{mm}-\mathrm{mrad}$ \\
Beam Total Energy & $8.89 \mathrm{GeV}$ \\
$\nu_{x}$ & 9.65 \\
$\nu_{y}$ & 9.694 \\
\hline
\end{tabular}

\subsection{Third Integer Resonance}

We discussed about the equations of motion for the third integer resonance in Chapter 2. and the fixed points of separatrix are given by equations 2.85 and 2.86 , from which we can say that

$$
\text { Size of separatrix } \propto \frac{\delta}{A}
$$

where $\delta$ is tune difference from the third integer tune and A depends on the sextupole strengths. To extract particles at a controlled pace, the size of the separatrix needs to be adiabatically reduced, which can be achieved by increasing the sextupole strengths or reducing the tune difference. The above relation clearly shows that to reduce the size of separatrix to zero, either $A$ (sextupole strengths) has to be increased to $\infty$ (which is impossible), or $\delta$ (tune difference) has to go to zero. Although changing tune gives you better control over extraction, it alters the beta function, thereby changing the orientation of the separatrix 
which alters the slope at which the particles are extracted. We can use a combination of changing the strength and tune to achieve reasonably good extraction.

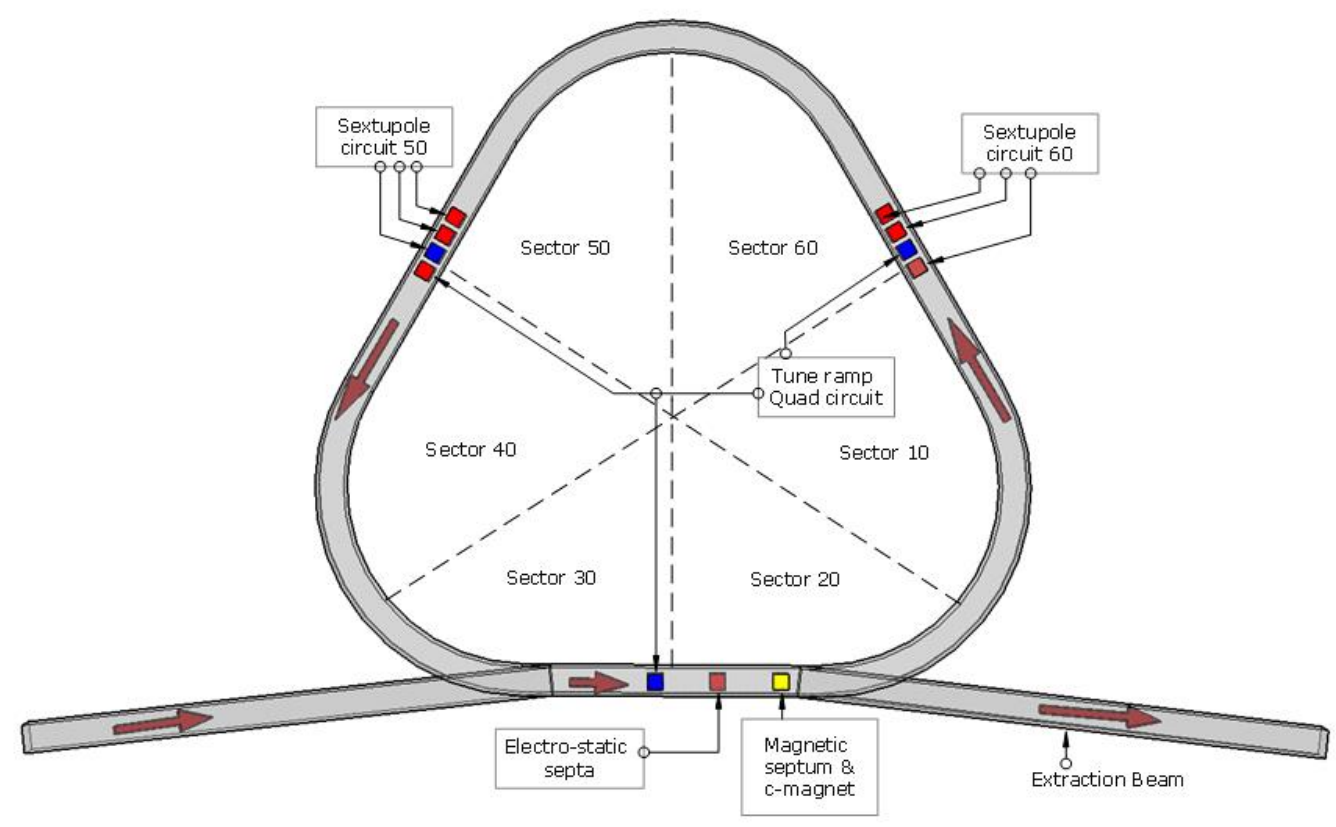

Figure 3.2: Sextupoles Placement

Placement of sextupoles and quadrupoles (which are used to change the tune) in the $\mathrm{DR}$ are shown in Figure 3.2. The phase evolution in both $\mathrm{R}$ and MAD-X can be seen in Figure 3.3. R simulation (Appendix C) which gives the approximate particle tracking is shown on the left, while MAD-X that can simulate to the high orders is shown on the right. We choose to run until 5th order because of computational limitations. Parameters for the extraction are given in the Table 3.2 
Table 3.2: Parameters that are considered for extraction [2]

\begin{tabular}{|c||c|}
\hline Type & value \\
\hline \hline Septum placement (from center of beam) & $12 \mathrm{~mm}$ \\
Septum thickness & $50 \mu \mathrm{m}$ \\
Spill Duration & $54 \mathrm{msec}(32000$ turns $)$ \\
Target extraction Rate & $0.003 \%$ \\
\hline
\end{tabular}
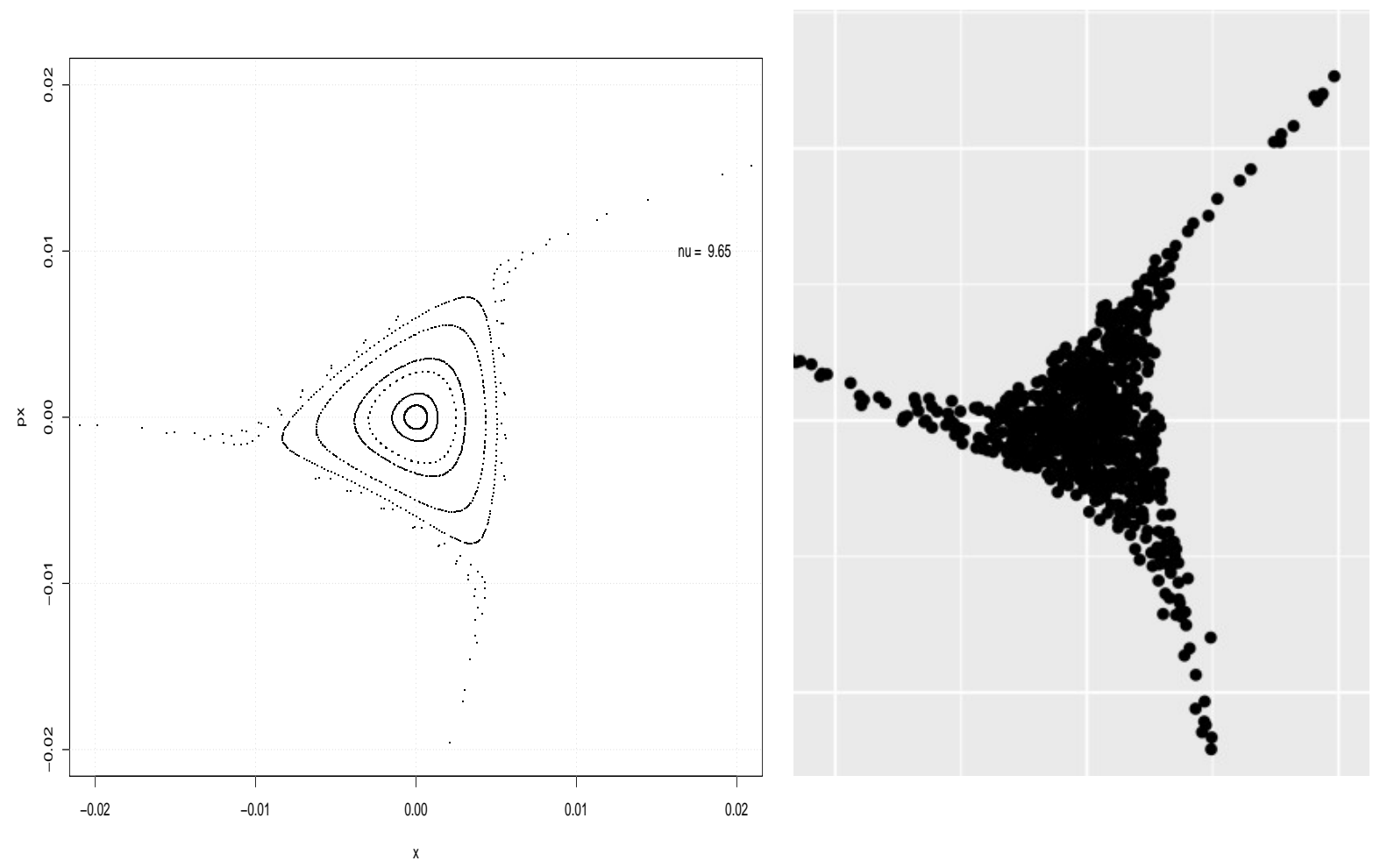

Figure 3.3: Phase evolution in R (on the Left) and MAD-X (on the Right)

\subsection{Noise extraction}

In order to accurately extract particles, noise can be included as a parameter to extract particles. By using a noise source to increase the beam emittance while keeping the separatrix 
configuration constant, a better control of extraction rate is expected. Earlier we discussed about how to use both sextupoles and tuning quadrupoles to achieve extraction. Noise is induced through an RF kicker to slowly increase the emittance, thereby pushing particles out of the separatrix. In the experiment a kicker magnet driven by low-amplitude white noise source will be utilized to generate random kicks. This will affect the emittance.

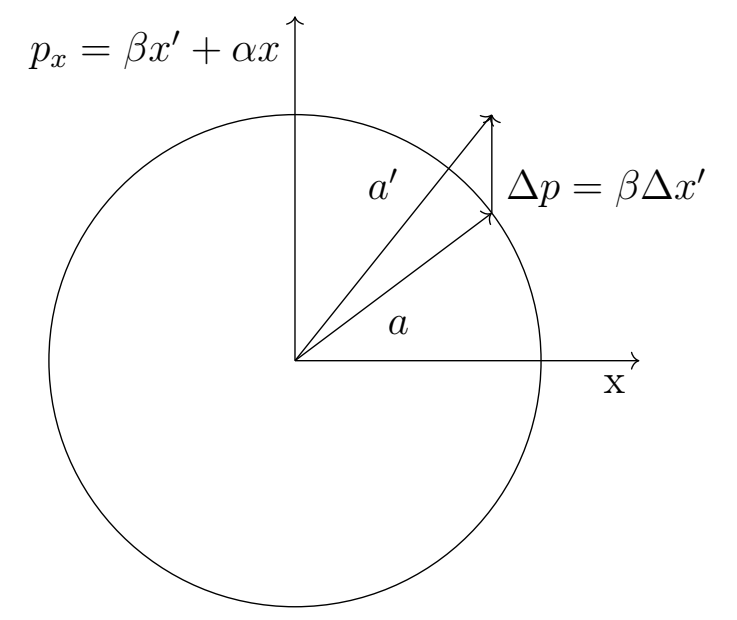

Figure 3.4: Noise introduction in Phase space

The area of the normalized phase space for an ensemble of particles is

$$
\begin{gathered}
\hat{\epsilon}=\beta \epsilon=\pi\left\langle x^{2}\right\rangle \\
\hat{\epsilon}=\pi \frac{\left\langle a^{2}\right\rangle}{2}
\end{gathered}
$$

The change of amplitude due to the noise of $\Delta x^{\prime}$ in each turn

$$
\begin{gathered}
\left\langle a^{2}\right\rangle=\left\langle a_{0}^{2}\right\rangle+\left\langle\Delta p_{x}^{2}\right\rangle-2\left\langle a_{0} \Delta p_{x} \cos (\text { ang })\right\rangle \\
\Delta\left\langle a^{2}\right\rangle=\left\langle\Delta p_{x}^{2}\right\rangle
\end{gathered}
$$


The change of the phase space area per turn

$$
\Delta \hat{\epsilon}=\pi \frac{\left\langle\Delta p_{x}^{2}\right\rangle}{2}=\pi \frac{\beta_{k}^{2}}{2}\left(\Delta x_{r m s}^{\prime}\right)^{2}
$$

where $\beta_{k}$ is $\beta$ value at the location of kicker device.

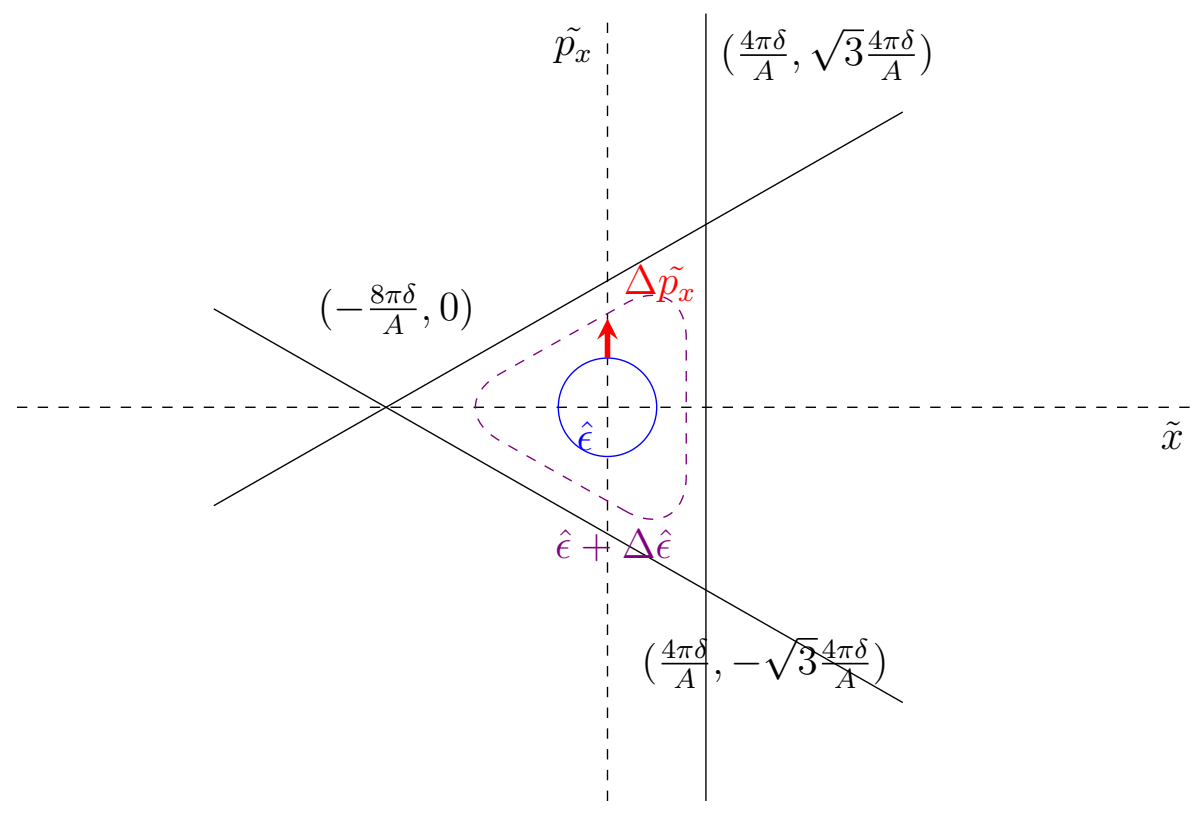

Figure 3.5: Emittance change due to noise

One can clearly see that the noise pushes particles closer to the boundaries of the separatrix. Our aim is to increase the emittance such that we extract particles at a constant rate. Results on how various parameters effect the rate of extraction are shown in the next chapter. 


\section{CHAPTER 4 \\ RESULTS AND CONCLUSION}

\subsection{Results}

Ultimately our goal is to simulate the particle tracking and beam extraction to see if we could extract the protons out of Delivery Ring (DR) at a constant rate towards the target. The following scenarios were investigated.

- Simulating the third integer resonance extraction varying Sextupoles, Quadrupoles for tune separately and combined on Delivery Ring lattice, this was repeated for fifty iterations and lot more test runs to get the parameters close to the actual experiment.

- Simulating the effects of noise on extraction. A simple R function is used to simulate random noise generated by a RF kicker. This was repeated multiple iterations until the extraction rate was close to the required value.

- Simulating the particle loss and the power loss due to particles hitting the septum foils during extraction.

Computations are performed using the Gaea [38] cluster at Northern Illinois University. $\mathrm{R}$ script was used to generate random noise and conditional logic is used to analyze the particle loss at the extraction point. Both $\mathrm{R}$ and MAD-X are used for tracking while bash script is used to vary the parameters at each turn. Tests were started with a sample size of 100 particles and 100 turns to serve as prototype for the analysis, slowly the sample size increased to $10^{5}$ particles and particle tracking time was increased to 54 msec (32000 turns). 
Run time for the simulation ranged from $30 \mathrm{~s}$ to $60 \mathrm{hrs}$ depending on the sample size and tracking time. For calculating power loss while extraction we assume all the particles having same kinetic energy $(8 \mathrm{GeV})$. Following analysis shows what would be the power loss for the design values of the experiment during extraction.

$$
\begin{aligned}
& \text { Energy of each particle }=1.424 \times 10^{-9} \mathrm{~J} \\
& \text { Total number of particles in the Delivery Ring }=1 \times 10^{12}
\end{aligned}
$$

According to Technical Design Report 2] the loss is expected to be less than 2\%. For $2 \%$ loss,

$$
\begin{aligned}
& \text { Total particle loss }=2 \times 10^{10} \\
& \text { Total energy loss }=28.48 \mathrm{~J} \\
& \text { Total power loss }=527 \mathrm{~W}
\end{aligned}
$$

\subsubsection{Interpreting Results}

Figures 4.1 through 4.6 show the simulation results for 5000 turns by varying different parameters. In all these figures, the green line represents tune of DR and yellow line represents strength of the sextupoles, both were varied from their initial value ( 0 in scale) to the final value (1 in scale). Turn numbers over which tune and sextupole strengths are changing were mentioned on the title of the figures. Target extraction rate (0.003 percent per turn) is represented by the pink line and the white line represents the trend line of the extraction rate when the noise is induced. 
While all the values shown in the plots are calculated based on simulation sample size ( $10^{5}$ particles), the power loss is calculated for original beam size used for the experiment (10 12 particles) by assuming that the following relation is true.

$$
\text { Power loss in Plot }=\text { Power loss for } 10^{5} \text { particles } \times \frac{10^{12}}{10^{5}}
$$

The plots are divided into two sections, with the one on left representing the extraction without noise when the tune and sextupole strengths are changing while the one on right represents the extraction process once the tune and sextupole strengths reached their final values and noise is introduced at each turn. For example, if a random noise of $\Delta x^{\prime}=10^{-6}$ is introduced starting from turn 1000 it means for each particle in the ensemble a random kick between $\Delta x^{\prime}= \pm 10^{-6}$ is given at every turn after 1000 .

Figure 4.1: Tune 0-250; Sextupole 0-500; Noise $\left(\Delta x^{\prime}\right)=10^{-6}$
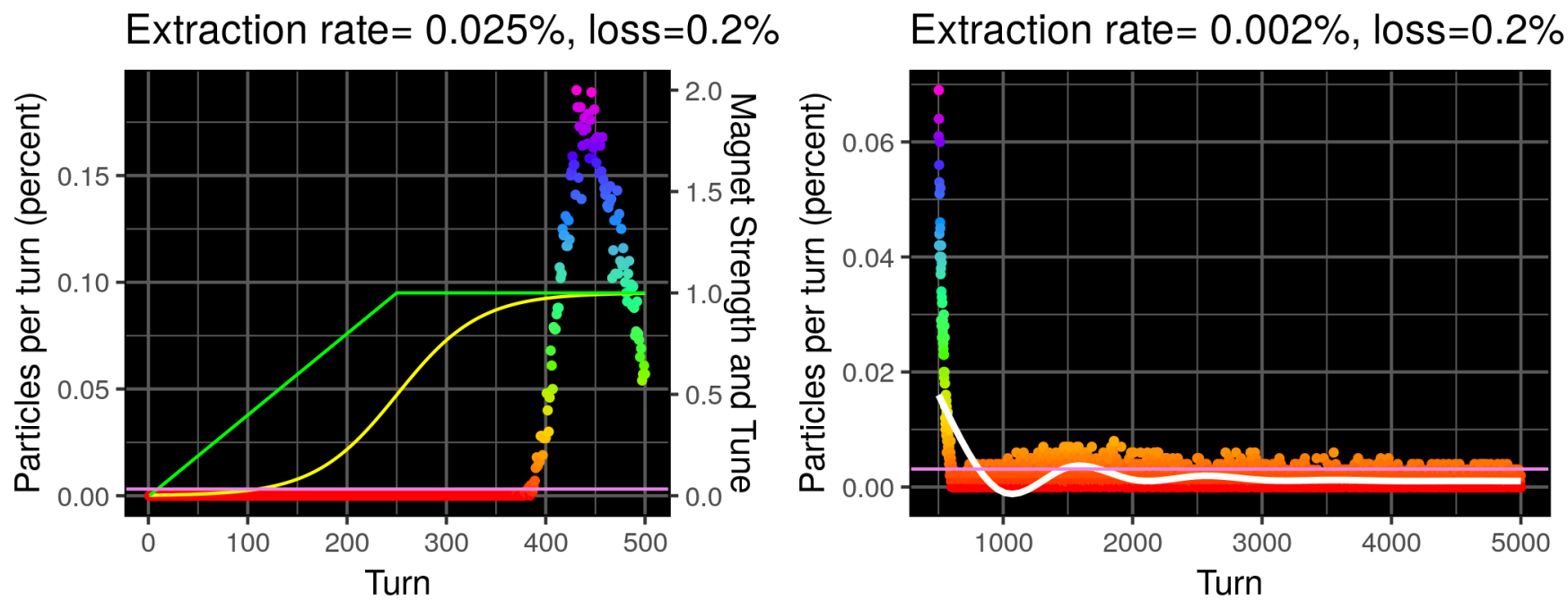

For 5000 turns extraction rate $-0.004 \%$, loss $-0.4 \%$ \& Power loss $-649 \mathrm{~W}$ 
Figure 4.2: Tune 0-250; Sextupole 0-500; Noise $\left(\Delta x^{\prime}\right)=5 \times 10^{-6}$

Extraction rate $=0.024 \%$, loss $=0.2 \% \quad$ Extraction rate $=0.011 \%$, loss $=0.8 \%$
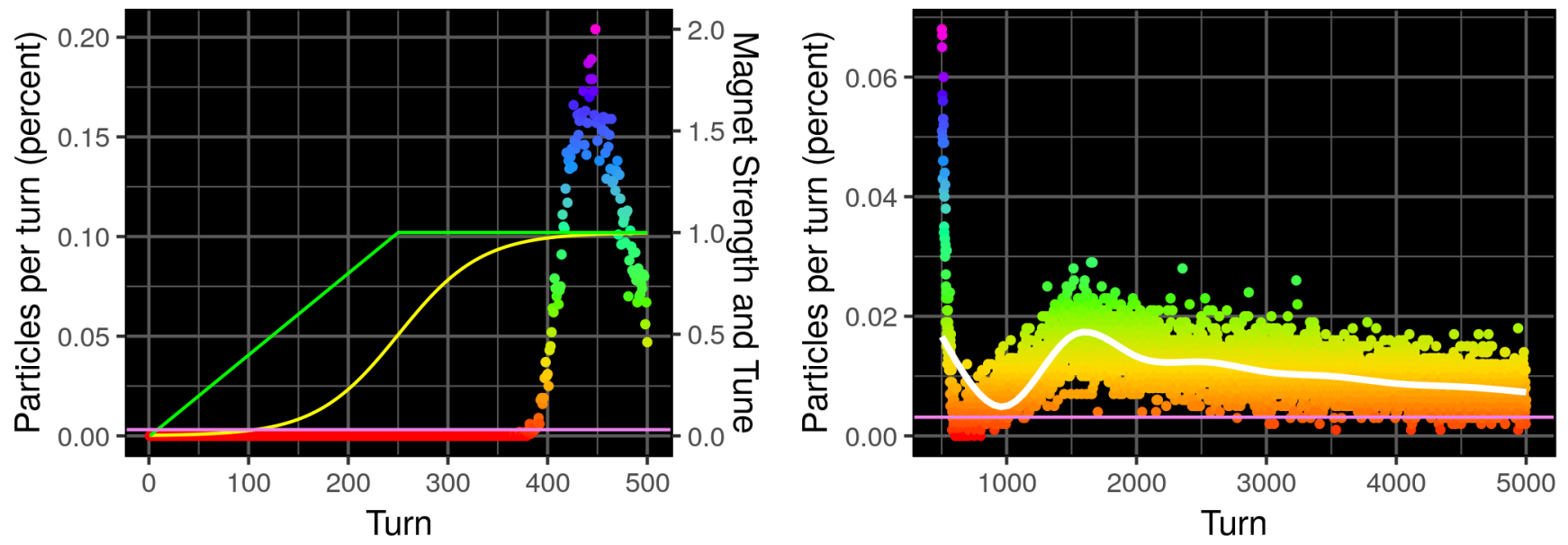

For 5000 turns extraction rate $-0.012 \%$, loss $-1.1 \%$ \& Power loss $-1765 \mathrm{~W}$

Figure 4.3: Tune 0-250; Sextupole 0-500; Noise $\left(\Delta x^{\prime}\right)=8 \times 10^{-6}$

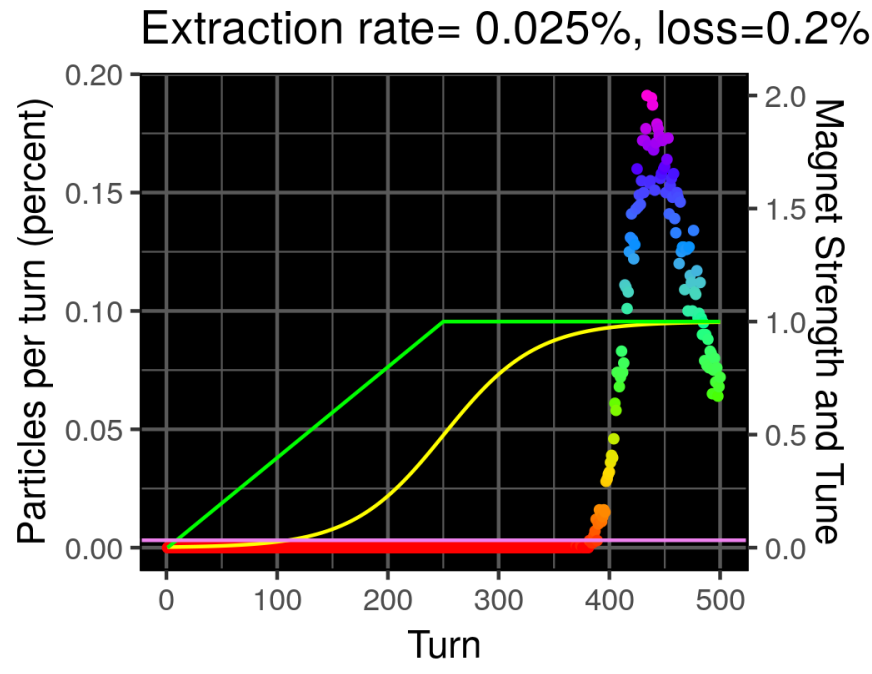

Extraction rate $=0.016 \%$, loss $=1.3 \%$

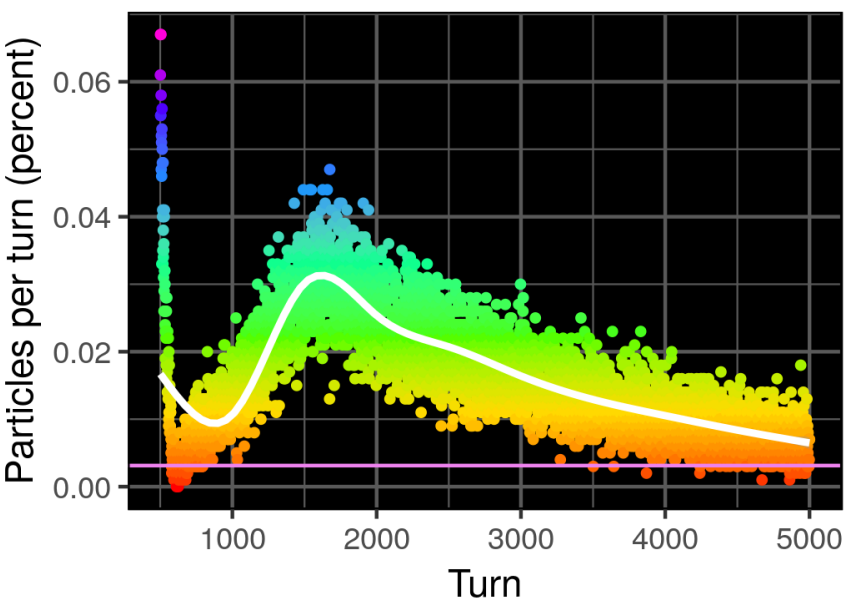

For 5000 turns extraction rate $-0.017 \%$, loss $-1.6 \%$ \& Power loss $-2612 \mathrm{~W}$ 
Figure 4.4: Tune 0-250; Sextupole 0-500; Noise $\left(\Delta x^{\prime}\right)=2 \times 10^{-6}$

Extraction rate $=0.024 \%$, loss $=0.2 \%$

Extraction rate $=0.004 \%$, loss $=0.3 \%$
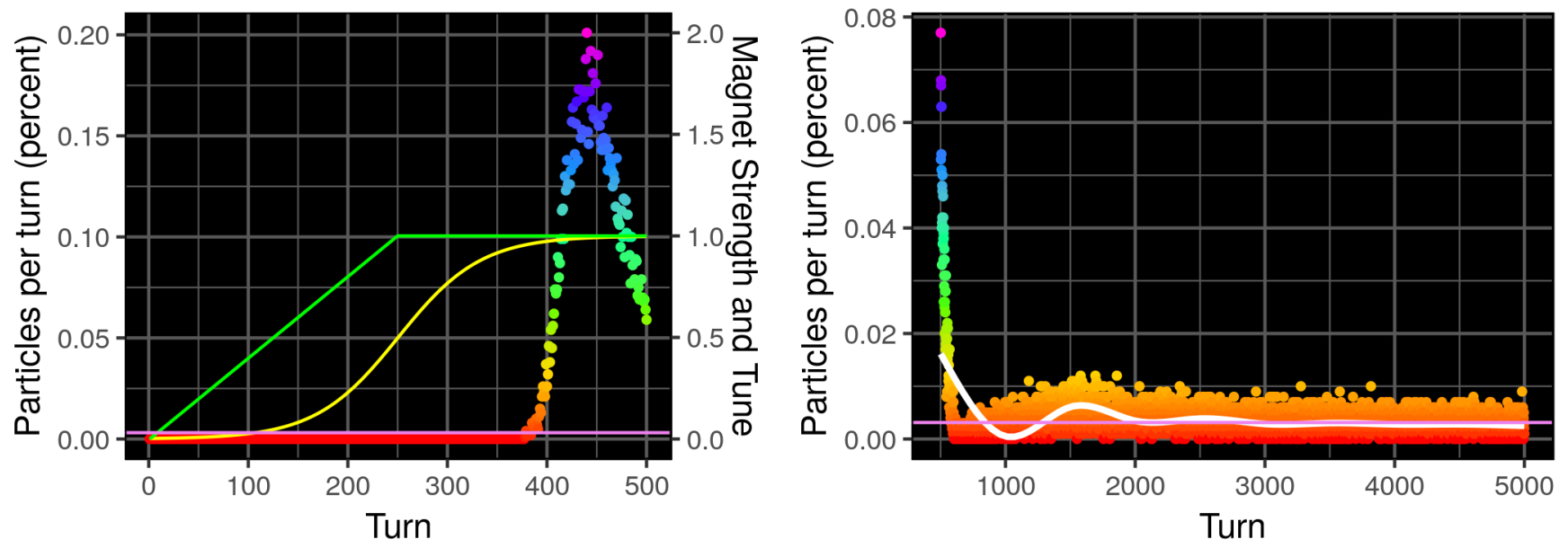

For 5000 turns extraction rate $-0.006 \%$, loss $-0.5 \%$ \& Power loss $-879 \mathrm{~W}$

Figure 4.5: Tune 0-1000; Sextupole 0-1000; Noise $\left(\Delta x^{\prime}\right)=2 \times 10^{-6}$

Extraction rate $=0.014 \%$, loss $=0.3 \% \quad$ Extraction rate $=0.004 \%$, loss $=0.3 \%$
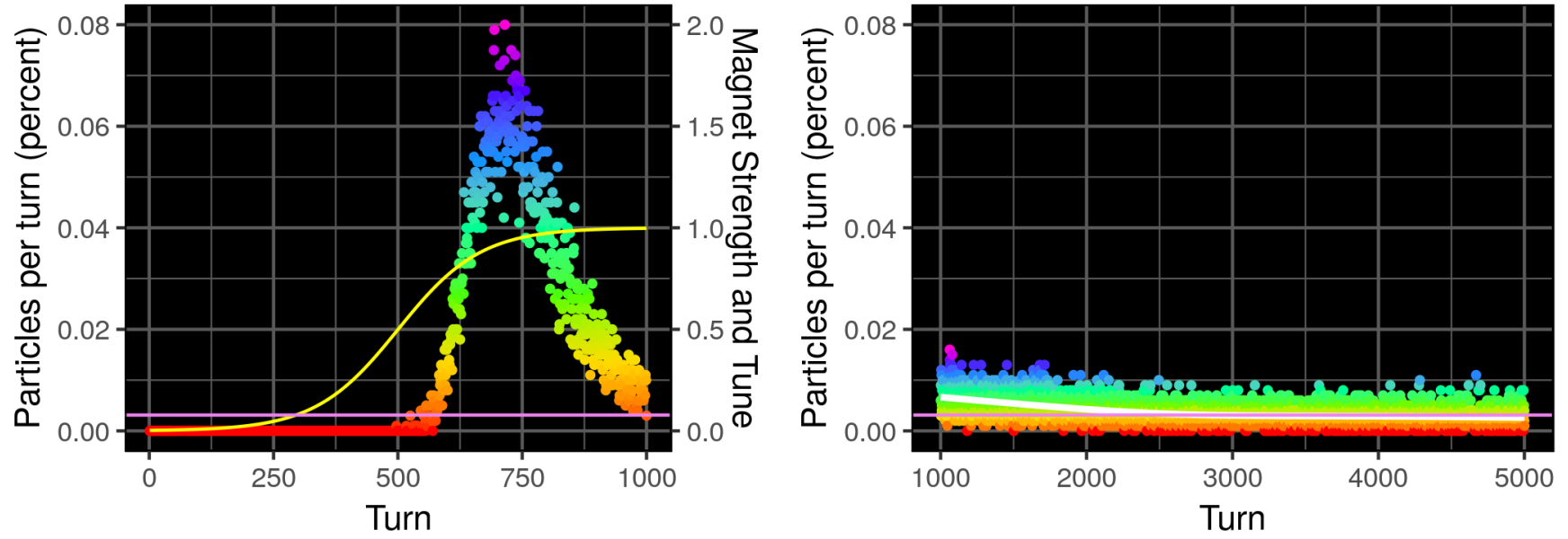

For 5000 turns extraction rate $-0.006 \%$, loss $-0.6 \%$ \& Power loss $-965 \mathrm{~W}$ 
Figure 4.6: Tune 0-2000; Sextupole 0-2000; Noise $\left(\Delta x^{\prime}\right)=2 \times 10^{-6}$
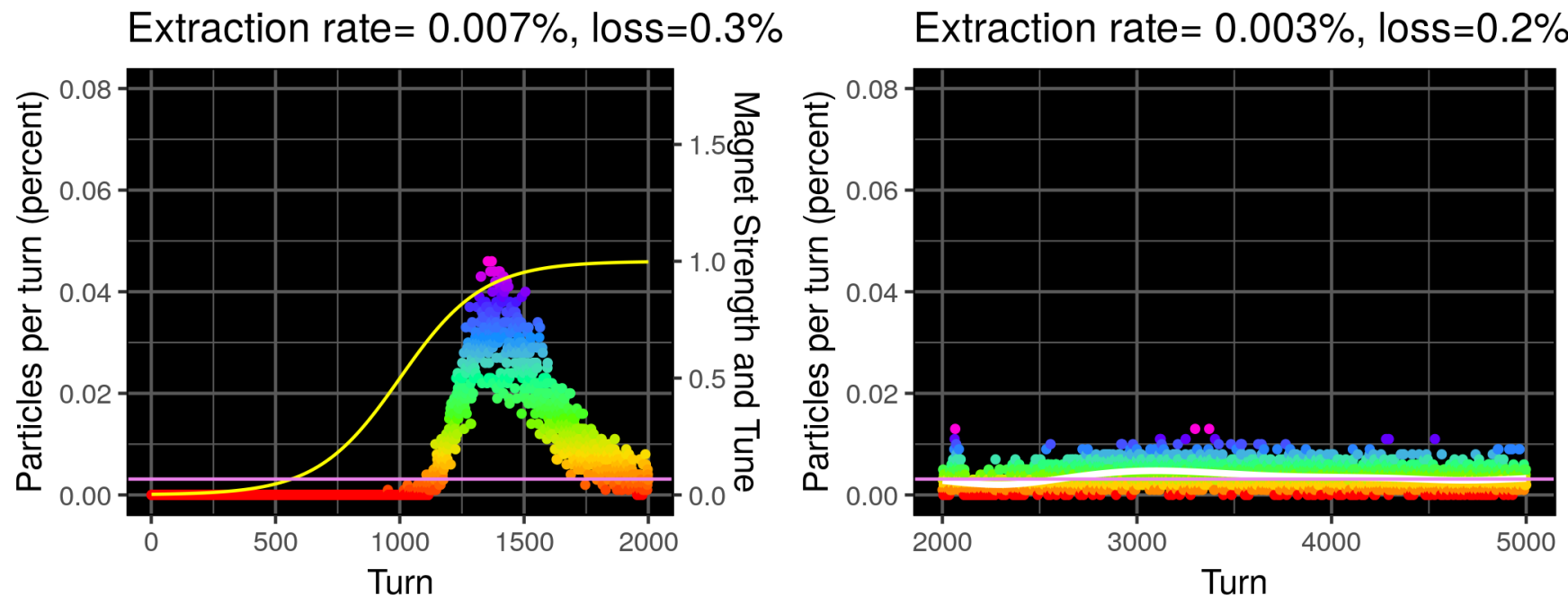

For 5000 turns extraction rate $-0.005 \%$, loss $-0.5 \%$ \& Power loss $-842 \mathrm{~W}$

As one can see from the figures, there is a transient peak of extracted particles just as the tune and sextupole strengths reach their final values which gives a much higher rate of extraction than required for a time period of about 500 turns. The desired extraction rate with minimum loss of particles is observed when the parameters are changed gradually and the closest extraction rate to the desired value without much losses is observed when tune and sextupoles are varied gradually over 2000 turns and the noise is $\Delta x^{\prime}=2 \times 10^{-6}$ as shown in Figure 4.6. While the extraction rate in the plots are per turn, the loss is cumulative for the number of turns in the plot associated with it. The power loss in the figures specify the value extrapolated for $10^{12}$ particles assuming the worst case scenario where all those hitting the septum foil [2] at the extraction location lose their entire energy. 


\subsection{Future directions}

Expanding the understanding of noise induced resonance extraction for variable energy levels of the beam and including the longitudinal parameters like momentum spread and dispersion will be important when we turn our attention to future plans for the accelerator complex at Fermilab in which the idea of varying the beam energy in the Delivery Ring will be examined. For example, if the beam energy is $2 \mathrm{GeV}$ instead of $8 \mathrm{GeV}$, then the beam size is bigger so the separatrix size has to be reconfigured to fit the new beam.

It is also appropriate to examine the ways to improve the target and detector system to handle higher intensities and better sensitivities.

\subsection{Conclusions}

The purpose of this study is to understand the motivations behind the search for Mu2e and the workings of how to deliver the beam with required intensity to make the experiment a reality. Utilizing the parameters from $M u 2 e$, understanding the beam dynamics, phase space evolution, and extraction have been the central part of this study.

Delivering the beam at right intensity is important to achieve high sensitivities in the search for CLFV and BSM through the Mu2e experiment. Simulations clearly show that particle's energy, beam emittance, tune of the machine, placement of the sextupoles and tuning quadrupoles, nature and magnitude of the noise induced, extraction location and phase space parameters at the extraction location influence the rate of extraction. Important

observation noted during simulation is that while using the resonance condition in magnets help extract the particles within the desired range of phase space values, the rate of extraction 
and thereby the outcoming intensity of the beam can be fine-tuned by using RF noise in addition to the magnets. 


\section{REFERENCES}

[1] G. Altarelli. The Higgs and the Excessive Success of the Standard Model, arXiv:1407.2122 [hep-ph], 2014.

[2] L. Bartoszek, et al, Mu2e Technical Design Report. arXiv:1501.05241 [physics.ins-det]

[3] MissMJ/PBS NOVA/Fermilab/Particle Data Group, Accessed from: https://en. wikipedia.org/wiki/File:Standard_Model_of_Elementary_Particles.svg.

[4] T. Toshiyuki. Super-Kamiokande collaboration, Super-Kamiokande atmospheric neutrino results, arXiv:0105023v1 [hep-ex], 2001.

[5] M. Tanabashi et al. Review of Particle Physics, Particle Data Group, Phys. Rev. D 98, 030001, 2018.

[6] L. Calibbi and G. Signorelli. Charged Lepton Flavour Violation: An Experimental and Theoretical Introduction, arXiv:1709.00294v2 [hep-ph], 2017.

[7] S. P. Martin Phenomenology of Particle Physics, http://www.niu.edu/spmartin/ phys686.

[8] S. P. Martin A Supersymmetry Primer, arXiv:9709356v7 [hep-ph], 2016.

[9] J. D. Wells PeV-Scale Supersymmetry, arXiv:9709356v7 [hep-ph], 2016.

[10] W. Altmannshofer, R. Harnik, J. Zupan. Low energy probes of PeV scale sfermions, arXiv:1308.3653v4 [hep-ph], 2016. 
[11] C. Csáki. The Minimal Supersymmetric Standard Model, arXiv:9606414v1 [hep-ph], 1996.

[12] H. P. Niles. Phenomenological Aspects of Supersymmetry, arXiv:9511313v1 [hep-ph], 1995.

[13] A. Rossi. Supersymmetric Seesaw without Singlet Neutrinos: Neutrino Masses and Lepton-Flavor Violation, arXiv:0207006v2 [hep-ph], 2002.

[14] A. M. Baldini et al. Search for the Lepton Flavor Violating Decay $\mu^{+} \longrightarrow e^{+} \gamma$ with the Full Dataset of the MEG Experiment, arXiv:1605.05081v3 [hep-ex], 2016.

[15] A. M. Baldini et al. The design of the MEG II experiment, arXiv:1801.04688v1 [physics.ins-det], 2018.

[16] N. M. Truong, DeeMe Collaboration. Search for $\mu \longrightarrow$ e conversion with DeeMe experiment at J-PARC MLF, PoS (FPCP2015) 060.

[17] A. K. Perrevoort, Mu3e Collaboration. Status of the Muße Experiment at PSI, arXiv: 1605.02906v1 [physics.ins-det], 2016.

[18] D. Bryman et al. The COMET Collaboration. An Experimental Search for Lepton Flavor Violating $\mu^{-} \longrightarrow e^{-}$Conversion at Sensitivity of $10^{16}$ with a Slow-Extracted Bunched Proton Beam, Accessed from: http://comet.kek.jp/Documents_files/ main-proposal.pdf.

[19] G. Pezzullo. The Mu2e experiment at Fermilab: a search for lepton flavor violation, arXiv:1705.06461v2 [hep-ex], 2017.

[20] A. Keshavarzi. Muon $g-2$ collaboration. The Muon $g-2$ experiment at Fermilab, arXiv:1905.00497v2 [hep-ex], 2019. 
[21] V. Cirigliano et al. Spin-dependent $\mu \rightarrow e$ conversion, arXiv:1703.02057v3 [hep-ph], 2018.

[22] Fermi National Accelerator Laboratory, U.S. Department of Energy. Accessed from: https://fnal.gov/pub/science/particle-accelerators/accelerator-complex. html.

[23] E. J. Prebys, S. Werkema. Fermi National Laboratory, Batavia, IL USA. Out of Time Beam Extinction in the Mu2e Experiment, FERMILAB-CONF-15-166-APC, Accessed from: http://lss.fnal.gov/archive/2015/conf/fermilab-conf-15-166-apc.pdf

[24] R. H. Bernstein. The Mu2e Experiment arXiv:1901.11099 [physics.ins-det], 2019.

[25] Mu2e Collaboration, Fermi National Accelerator Laboratory, U.S. Department of Energy. Accessed from: https://mu2e.fnal.gov/images_v2/.

[26] D. A. Edwards and M. J. Syphers. An Introduction to the Physics of High Energy Accelerators. Wiley VCH, Weinheim, Germany, 2004.

[27] J. D. Jackson. Classical Electrodynamics. Wiley, New York, USA, 3rd ed. edition, 1999.

[28] S. M. Carroll. Spacetime and geometry. An introduction to general relativity. Addison Wesley, San Francisco, USA, 2004.

[29] P. Barnes et al. Birbeck college, University of London. Accessed from: http://pd. chem.ucl.ac.uk/pdnn/inst2/work.htm.

[30] X. C. Vidal and R. C. Manzano, Santiago, Spain. Accessed from: https://www. lhc-closer.es/taking_a_closer_look_at_lhc/0.magnetic_multipoles.

[31] A. Wolski, University of Liverpool. Accessed from: http:http://hep.ph.liv.ac.uk/ hock/Good_References/Good_References.html. 
[32] E. D. Courant and H. S. Snyder. Theory of the alternating-gradient synchrotron. Annals of Physics, 3(1), 1-48, 1958.

[33] Helmut Wiedmann. Particle Accelerator Physics. Springer International Publishing, Switzerland, 2015.

[34] Klaus Floettmann Some basic features of the beam emittance Physical Review, Special Topics - Accelerators and Beams, Volume 6, 034202 (2003). https://link.aps.org/ doi/10.1103/PhysRevSTAB.6.034202.

[35] RStudio Team (2016). RStudio: Integrated Development for R. RStudio, Inc., Boston, MA http://www.rstudio.com/.

[36] European Organization for Nuclear Research. MAD-X(Methodical Accelerator Design) Version 5.02.00. [Computer software], 1990. Accessed from: http://madx.web.cern. $\operatorname{ch} / \operatorname{madx}$.

[37] Fermi National Accelerator Laboratory, U.S. Department of Energy. Accessed from: http://operations.fnal.gov/members/baginski/drawings/pbar-map-new.pdf.

[38] Gaea: High performance computing cluster, Northern Illinois University. Accessed from: http://www.cs.niu.edu/facilities/index.shtml 
APPENDIX A

HAMILTONIAN IN ACCELERATOR COORDINATES 
This appendix gives a detailed derivation for the equations that are discussed in Chapter 2 . Starting from the Hamiltonian in Eq. 2.7

$$
H=\sqrt{\left(m c^{2}\right)^{2}+(\vec{P}-e \vec{A})^{2} c^{2}}+e \Phi
$$

where $\vec{P}$ is canonical momentum, $\vec{A}$ is the vector potential and $\Phi$ is the scalar potential. The components of canonical momentum in the new accelerator system coordinate system are as follows:

$$
P_{x}=\vec{P} \cdot \hat{x} \quad P_{y}=\vec{P} \cdot \hat{y} \quad P_{s}=\vec{P} \cdot \hat{s}\left(1+\frac{x}{\rho}\right)
$$

Components of vector potential in new coordinate system are:

$$
A_{x}=\vec{A} \cdot \hat{x} \quad A_{y}=\vec{A} \cdot \hat{y} \quad A_{s}=\vec{A} \cdot \hat{s}\left(1+\frac{x}{\rho}\right)
$$

Hamiltonian is

$$
H=c \sqrt{(m c)^{2}+\left(P_{x}-e A_{x}\right)^{2}+\left(P_{y}-e A_{y}\right)^{2}+\frac{1}{(1+x / \rho)^{2}}\left(P_{s}-e A_{s}\right)^{2}}+e \Phi
$$

Hamiltonian equations of motion are,

$$
\begin{array}{ll}
\frac{\partial H}{\partial P_{x}}=\dot{x} & \frac{\partial H}{\partial x}=-\dot{P}_{x} \\
\frac{\partial H}{\partial P_{y}}=\dot{y} & \frac{\partial H}{\partial y}=-\dot{P}_{y} \\
\frac{\partial H}{\partial P_{s}}=\dot{s} & \frac{\partial H}{\partial s}=-\dot{P}_{s} .
\end{array}
$$


In these coordinates we differentiate with respect to path length instead of time, so we have [26]:

$$
x^{\prime}=\frac{d x}{d s}=\frac{d x}{d t} \frac{d t}{d s}=\frac{\partial H}{\partial P_{x}}\left(\frac{\partial H}{\partial P_{s}}\right)^{-1}
$$

Assuming At constant $\mathrm{H}$, using chain rule we have

$$
\begin{array}{r}
d H=\left(\frac{\partial H}{\partial P_{x}}\right)_{P_{s}} d P_{x}+\left(\frac{\partial H}{\partial P_{s}}\right)_{P_{x}} d P_{s}=0 \\
\frac{\partial P_{s}}{\partial P_{x}}=-\frac{\partial H}{\partial P_{x}}\left(\frac{\partial H}{\partial P_{s}}\right)^{-1}
\end{array}
$$

By substituting the above value we have,

$$
\begin{array}{r}
x^{\prime}=\frac{\partial\left(-P_{s}\right)}{\partial P_{x}} \\
P_{x}^{\prime}=\frac{d P_{x}}{d s}=\frac{\partial P_{x}}{d t} \frac{d t}{d s}=-\frac{\partial\left(-P_{s}\right)}{\partial x}
\end{array}
$$

Similarly,

$$
\begin{array}{r}
y^{\prime}=\frac{\partial\left(-P_{s}\right)}{\partial P_{y}} \quad P_{y}^{\prime}=-\frac{\partial\left(-P_{s}\right)}{\partial y} \\
t^{\prime}=\frac{\partial P_{s}}{\partial H} \quad H^{\prime}=-\frac{\partial P_{s}}{\partial t}
\end{array}
$$

Equations A.10 and A.11 look like Hamiltonian equations of motion if the Hamiltonian is $-P_{s}$ with the canonical variables being $\mathrm{x}, P_{x} ; \mathrm{y}, P_{y}$, and $\mathrm{t},-\mathrm{H}$ so rewriting the Hamiltonian we have,

$$
\frac{H-e \Phi}{c}=\sqrt{(m c)^{2}+\left(P_{x}-e A_{x}\right)^{2}+\left(P_{y}-e A_{y}\right)^{2}+\frac{1}{(1+x / \rho)^{2}}\left(P_{s}-e A_{s}\right)^{2}}
$$

with a little re-arrangement we have,

$$
\left(\frac{P_{s}-e A_{s}}{1+x / \rho}\right)^{2}=\left(\frac{H-e \Phi}{c}\right)^{2}-(m c)^{2}-\left(P_{x}-e A_{x}\right)^{2}-\left(P_{y}-e A_{y}\right)^{2}
$$




$$
P_{s}=\sqrt{\left[\left(\frac{H-e \Phi}{c}\right)^{2}-(m c)^{2}-\left(P_{x}-e A_{x}\right)^{2}-\left(P_{y}-e A_{y}\right)^{2}\right]\left(1+\frac{x}{\rho}\right)^{2}}+e A_{s}
$$

By taking the new canonical variables into consideration, we have the new Hamiltonian as $\mathscr{H}=-P_{s}$

$$
\mathscr{H}=-\sqrt{\left[\left(\frac{H-e \Phi}{c}\right)^{2}-(m c)^{2}-\left(P_{x}-e A_{x}\right)^{2}-\left(P_{y}-e A_{y}\right)^{2}\right]\left(1+\frac{x}{\rho}\right)^{2}}-e A_{s}
$$

\section{A.1 Assumptions}

A few assumptions we can make for our case(just to make our life easier) are as follows

- 2D magnetic field, $\vec{B}=B_{x} \hat{x}+B_{y} \hat{y}$, which means we can assume vector potential with just z component and $A_{x}=A_{y}=0$

- No Electric Field $(H-e \Phi)$ can be written just as $H$

- Constant Energy which means

$$
\left(\frac{H}{c}\right)^{2}-(m c)^{2}=\vec{P}^{2}
$$

Hamiltonian is

$$
\mathscr{H}=-\sqrt{\left(\vec{P}^{2}-P_{x}^{2}-P_{y}^{2}\right)\left(1+\frac{x}{\rho}\right)^{2}}-e A_{s}
$$

Let $P_{0}$ be the momentum of the particle on reference orbit and $\vec{P}=P_{0}(1+\delta)$

$$
\tilde{\mathscr{H}}=\frac{\mathscr{H}}{P_{0}}=-\sqrt{\left(\frac{\vec{P}^{2}}{P_{0}^{2}}-\frac{P_{x}^{2}}{P_{0}^{2}}-\frac{P_{y}^{2}}{P_{0}^{2}}\right)\left(1+\frac{x}{\rho}\right)^{2}}-e \frac{A_{s}}{P_{0}}
$$


Let $\frac{P_{x}}{P_{0}}=\tilde{P}_{x}$ and $\frac{P_{y}}{P_{0}}=\tilde{P}_{y}$ then we have

$$
\begin{gathered}
\tilde{\mathscr{H}}=-\left(1+\frac{x}{\rho}\right) \sqrt{\left((1+\delta)^{2}-\tilde{P}_{x}^{2}-\tilde{P}_{y}^{2}\right)}-e \frac{A_{s}}{P_{0}} \\
\tilde{\mathscr{H}}=-\left(1+\frac{x}{\rho}\right)(1+\delta) \sqrt{\left(1-\frac{\tilde{P}_{x}^{2}}{(1+\delta)^{2}}-\frac{\tilde{P}_{y}^{2}}{(1+\delta)^{2}}\right)}-e \frac{A_{s}}{P_{0}}
\end{gathered}
$$

A couple more assumptions that suit our case

- Paraxial approximation $\tilde{P}_{x}, \tilde{P}_{y}<<1$

- Low momentum spread $\delta<<1$

Using the above assumptions we can expand the equation (24) with taylor series, we have

$$
\begin{gathered}
\tilde{\mathscr{H}}=-\left(1+\frac{x}{\rho}\right)(1+\delta)\left(1-\frac{\tilde{P}_{x}{ }^{2}}{2(1+\delta)^{2}}-\frac{\tilde{P}_{y}{ }^{2}}{2(1+\delta)^{2}}\right)-e \frac{A_{s}}{P_{0}} \\
\tilde{\mathscr{H}}=-\left(1+\frac{x}{\rho}\right)\left(1+\delta-\frac{\tilde{P}_{x}^{2}}{2(1+\delta)}-\frac{\tilde{P}_{y}^{2}}{2(1+\delta)}\right)-e \frac{A_{s}}{P_{0}} \\
x^{\prime}=\frac{\partial \mathscr{H}}{\partial P_{x}}=\frac{\tilde{P}_{x}}{1+\delta} \\
y^{\prime}=\frac{\partial \mathscr{H}^{2}}{\partial P_{y}}=\frac{\tilde{P}_{y}}{1+\delta}
\end{gathered}
$$

Using the above equations we can rewrite the Hamiltonian as follows

$$
\tilde{\mathscr{H}}=-\left(1+\frac{x}{\rho}\right)\left(1+\delta-\frac{x^{\prime 2}}{2}-\frac{y^{\prime 2}}{2}\right)-\frac{e}{P_{0}} \vec{A} \cdot \hat{s}\left(1+\frac{x}{\rho}\right)
$$


APPENDIX B

MATRIX REPRESENTATION OF COURANT-SNYDER PARAMETERS 
Using the general solution from Eq. 2.38 we can rewrite the hills equation as follows

$$
x^{\prime \prime}+K x=A\left(2 w^{\prime} \psi^{\prime}+w \psi^{\prime \prime}\right) \sin (\psi+\delta)+A\left(w^{\prime \prime}-w \psi^{\prime 2}+K w\right) \cos (\psi+\delta)=0
$$

multiplying the sine term by $w$ we have

$$
2 w w^{\prime} \psi^{\prime}+w^{2} \psi^{\prime \prime}=\left(w^{2} \psi^{\prime}\right)^{\prime}=0 \Longrightarrow \psi^{\prime}=\frac{k}{w(s)^{2}}
$$

where $\mathrm{k}$ is constant of integration. The general solution can also be rewritten [26] as,

$$
x=w(s)\left(A_{1} \cos \psi+A_{2} \sin \psi\right)
$$

and

$$
x^{\prime}=\left(A_{1} w^{\prime}+\frac{A_{2} k}{w}\right) \cos \psi+\left(A_{2} w^{\prime}-\frac{A_{1} k}{w}\right) \sin \psi
$$

using the initial conditions of $x_{0}, x_{0}^{\prime}$ constant values are,

$$
A_{1}=\frac{x_{0}}{w}, \quad A_{2}=\frac{x_{0}^{\prime} w-x_{0} w^{\prime}}{k}
$$

Assuming $w$ as a periodic function, the resulting matrix equation is

$$
\left(\begin{array}{c}
x \\
x^{\prime}
\end{array}\right)_{s_{0}+C}=\left(\begin{array}{cc}
\cos \Delta \psi_{C}-\frac{w w^{\prime}}{k} \sin \Delta \psi_{C} & \frac{w^{2}}{k} \sin \Delta \psi_{C} \\
-\frac{1+\left(w w^{\prime} / k\right)^{2}}{w^{2} / k} \sin \Delta \psi_{C} & \cos \Delta \psi_{C}+\frac{w w^{\prime}}{k} \sin \Delta \psi_{C}
\end{array}\right)\left(\begin{array}{c}
x \\
x^{\prime}
\end{array}\right)_{s_{0}}
$$


which we can rewrite by defining the Courant-Synder parameters we discussed in Equations 2.47, 2.48, 2.49 as follows,

$$
\left(\begin{array}{c}
x \\
x^{\prime}
\end{array}\right)_{s_{0}+C}=\left(\begin{array}{cc}
\cos \Delta \psi_{C}+\alpha \sin \Delta \psi_{C} & \beta \sin \Delta \psi_{C} \\
-\gamma \sin \Delta \psi_{C} & \cos \Delta \psi_{C}-\alpha \sin \Delta \psi_{C}
\end{array}\right)\left(\begin{array}{c}
x \\
x^{\prime}
\end{array}\right)_{s_{0}}
$$




\section{APPENDIX C}

\section{PARTICLE TRACKING IN R}


Following is the particle tracking simulation logic used in $\mathrm{R}$ (Only the main details of the code are included here).

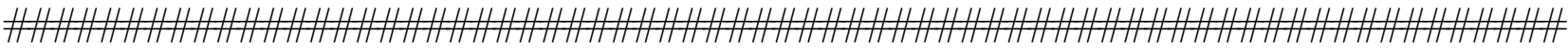

for ( i in $0:($ Nturns -1$))$

\{

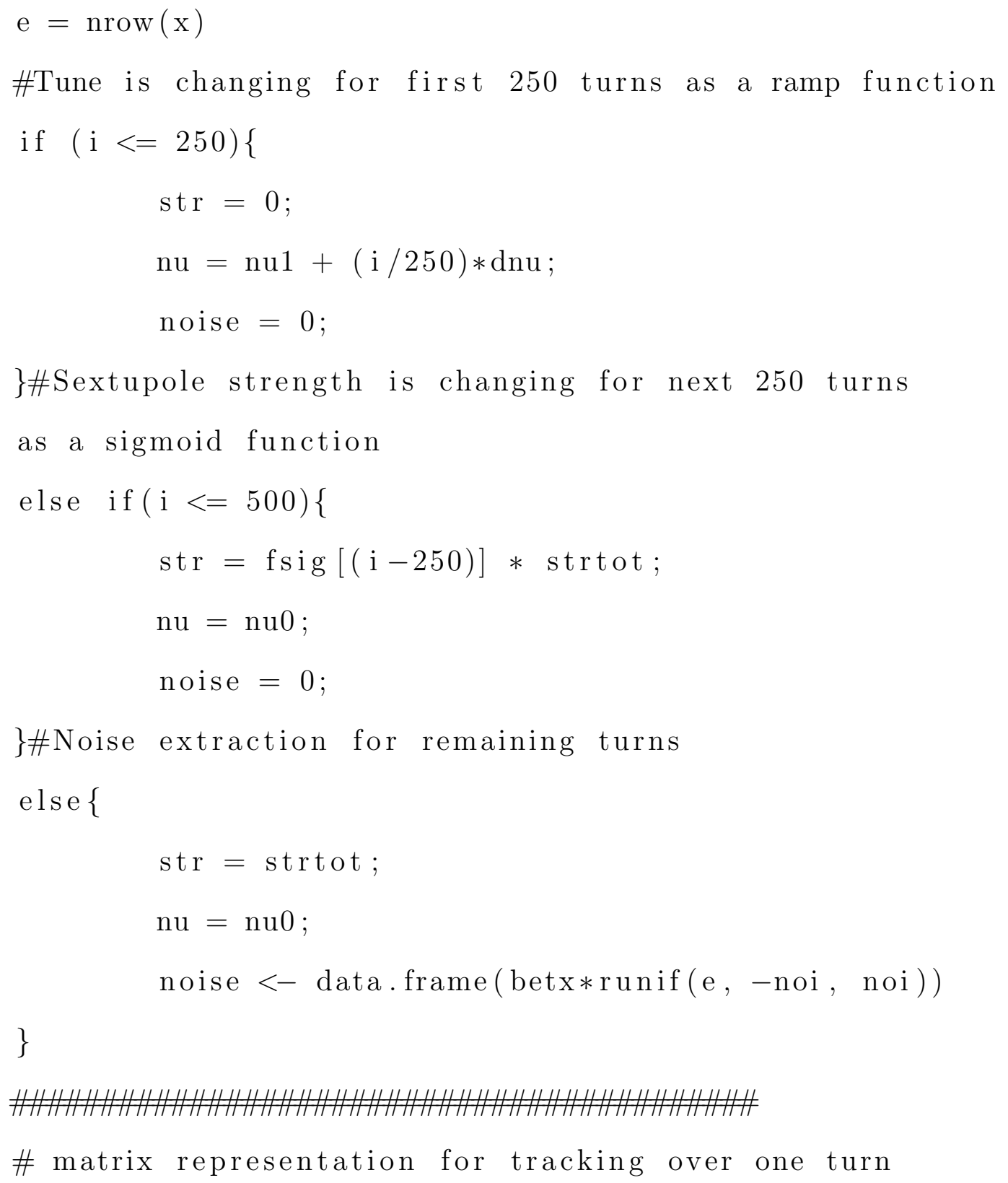



$\mathrm{a}<-\cos (2 * \mathrm{pi} * \mathrm{nu})$
$\mathrm{b}<-\sin (2 * \mathrm{pi} * \mathrm{nu})$
$\mathrm{c}<--\mathrm{b}$
$\mathrm{d}<-\mathrm{a}$

\#\#\#\#\#\#\#\#\#\#\#\#\#\#\#\#\#\#\#\#\#\#\#\#\#\#\#\#\#\#\#\#\#\#\#\#\#\#\#\#

\#Field perturbations

$\operatorname{dpx}<-\operatorname{betx} *\left(\operatorname{str} * x^{\wedge} 2\right) / 2$

px $1<-$ px - dpx + noise

$\mathrm{u} 1<-\mathrm{a} * \mathrm{x}+\mathrm{b} * \mathrm{px} 1$

$\mathrm{px} \quad<-\mathrm{c} * \mathrm{x} \quad+\mathrm{d} * \mathrm{px} 1$

$\mathrm{x}<-\mathrm{u} 1$

$\operatorname{xpx}<-$ data.frame $(\mathrm{x}, \mathrm{px})$

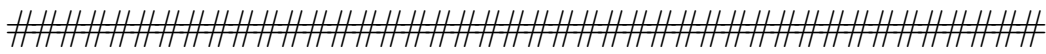

\#Turn and extraction data after each turn

tdata $=\operatorname{xpx}[$ which $(\operatorname{xpx}[1]>-0.012)$,

edata $=\operatorname{xpx}[$ which $(\operatorname{xpx}[1]<=-0.012)$,

if $($ nrow $($ edata $)=0)\{$

edata $<-$ data.frame $(0,0)$

\}

$\mathrm{x}<-\operatorname{tdata}[1]$

px $<-\operatorname{tdata}[2]$

turndata $<-$ data.frame $(\mathrm{i}+1$, tdata $)$

exdata $<-$ data.frame $(i+1$, edata $)$

\} 